\begin{tabular}{|c|l|}
\hline Title & Phase field model with a variable chemical potential \\
\hline Author(s) & Tonegawa, Y. \\
\hline Citation & Hokkaido University Preprint Series in Mathematics, 509, 1-30 \\
\hline Issue Date & 2000-12-1 \\
\hline DOI & 10.14943/83655 \\
\hline Doc URL & http://hdl.handle.net/2115/69259 \\
\hline Type & bulletin (article) \\
\hline File Information & pre509.pdf \\
\hline
\end{tabular}

Instructions for use 
PHASE FIELD MODEL WITH A VARIABLE CHEMICAL POTENTIAL

YOSHIHIRO TONEGAWA

Series \# 509. December 2000 


\section{HOKKAIDO UNIVERSITY \\ PREPRINT SERIES IN MATHEMATICS}

\#484 I. A. Bogaevski and G. Ishikawa, Lagrange mappings of the first open Whitney umbrella, 22 pages. 2000.

\#485 A. Arai and H. Kawano, A class of deformations of the Schrödinger representation of the Heisenberg commutation relation and exact solution to a Heisenberg equation and a Schrödinger equation, 22 pages. 2000.

\#486 T. Nakazi, Functions in $N_{+}$with the positive real parts on the boundary, 21 pages. 2000.

\#487 Y. Shibukawa, Classification of the $R$-operator, 36 pages. 2000.

\#488 A. İnoue, Asymptotic behaviour for partial autocorrelation functions of fractional ARIMA processes, 20 pages. 2000 .

\#489 S. Ohtani, Construction of unramified Galois extensions over maximal abelian extensions of algebraic number fields, 14 pages. 2000.

\#490 T. Nakazi and T. Yamamoto, The real part of an outer function and a Helson-Szegö weight, 13 pages. 2000 .

\#491 A. Yamagami, On Gouvêás conjecture on controlling the conductor, 11 pages. 2000.

\#492 I. Tsuda and M. Hatakeyama, Making sense of internal logic: Theory and a case study, 10 pages. 2000.

\#493 I. Tsuda, Towards an interpretation of dynamic neural activity in terms of chaotic dynamical systems, 73 pages. 2000 .

\#494 T. Mikami, Optimal control for absolutely continuous stochastic processes and the mass transportation problem, 17 pages. 2000.

\#495 M. Arisawa and Y. Giga, Anisotropic curvature flow in a very thin domain, 21 pages. 2000.

\#496 T. Nakazi, Backward shift invariant subspaces in the bidisc, 9 pages. 2000.

\#497 Y. Giga, K. Inui, J. Kato and S. Matsui, Remarks on the uniqueness of bounded solutions of the NavierStokes equations, 4 pages. 2000.

\#498 Y. Giga, M. Paolini and P. Rybka, On the motion by singular interfacial energy, 21 pages. 2000.

\#499 J. Escher and Y. Giga, On a limiting motion and self-interactions of curves moved by the intermediate surface diffusion flow, 12 pages. 2000.

\#500 I. Tsuda and S. Kuroda, Cantor coding in the hippocampus, 20 pages. 2000.

\#501 M. Tsujii, Fat solenoidal attractors, 20 pages. 2000.

\#502 A. Arai, Ground state of the massless Nelson model without infrared cutoff in a non-Fock representation, 19 pages. 2000.

\#503 Y. Giga, S. Matsui and O. Sawada, Global existence of two-dimensional Navier-Stokes flow with nondecaying initial velocity, 19 pages. 2000.

\#504 A. Inoue and Y. Kasahara, Partial autocorrelation functions of the fractional ARIMA processes with negative degree of differencing, 14 pages. 2000 .

\#505 T. Nakazi, Interpolation problem for $\ell^{1}$ and a uniform algebra, 12 pages. 2000.

\#506 R. Kobayashi and Y. Giga, On anisotropy and curvature effects for growing crystals, 38 pages. 2000.

\#507 A. Arai, Instability in the spectral and the Fredholm properties of an infinite dimensional Dirac operator on the abstract Boson-Fermion Fock space, 6 pages. 2000.

\#508 A. Arai, Supersymmetric methods for constructing soliton-type solutions to multi-component nonlinear Schrö dinger and Klein-Gordon equations, 22 pages. 2000. 


\title{
PHASE FIELD MODEL WITH A VARIABLE CHEMICAL POTENTIAL
}

\author{
YOSHIHIRO TONEGAWA
}

\begin{abstract}
We study some asymptotic behavior of phase interfaces with variable chemical potential under the uniform energy bound. The problem is motivated by the Cahn-Hilliard equation, where one has a control of the total energy and chemical potential. We show that the limit interface is an integral varifold with generalized $L^{p}$ mean curvature. The convergence of interfaces as $\varepsilon \rightarrow 0$ is in the Hausdorff distance sense.
\end{abstract}

\section{INTRODUCTION}

In this paper, we study some asymptotic behavior of phase interfaces in the van der Waals-Cahn-Hilliard theory of phase transitions. The equation we consider is

$$
-\varepsilon \Delta u+\frac{W^{\prime}(u)}{\varepsilon}=f
$$

where $u: U \subset \mathbb{R}^{n} \rightarrow \mathbb{R}, n \geq 2$, is the normalized density distribution of a two-phase fluid and $W$ is a double well potential with strict local minima at \pm 1 . The function $f$ may be cosidered as a chemical potential field in the two-phase fluid model ([23]) and $\varepsilon \approx 0$ is a parameter which gives the order of interface thickness.

The associated energy,

$$
E_{\varepsilon}(u)=\int_{\Omega} \frac{\varepsilon}{2}|\nabla u|^{2}+\frac{W(u)}{\varepsilon},
$$

and the behavior of minimizers of this energy with a volume constraint, were initially studied in $[30,39]$ within the framework of $\Gamma$-convergence ([16]) and subsequently generalized by many authors $([6,20,26,31$, $35,40])$. In this case, the minimizers satisfy the equation (1.1) with some constant $f \equiv c$, and the functional $E_{\varepsilon}(\cdot) \Gamma$-converges to the area functional. The limit interface as $\varepsilon \rightarrow 0$ is area-minimizing with a given volume constraint. It was also proved ([27]) that the constant mean

1991 Mathematics Subject Classification. Primary: 49Q20; Secondary: 35J60, $80 \mathrm{~A} 22,82 \mathrm{~B} 26$. 


\section{YOSHIHIRO TONEGAWA}

curvature of the limit interface is determined by the chemical potential and $\sigma=\int_{-1}^{1} \sqrt{W(s) / 2} d s$.

In [24], we studied the behavior of general critical points of the functional (1.2) with a volume constraint and showed that the interface is close in the Hausdorff distance sense to a locally constant mean curvature hypersurface when $\varepsilon \approx 0$. This corresponds to studying (1.1) again with constant $f \equiv c$, with suitable bounds on the energy and c. Note that due to the non-convexity of the functional, there can be solutions which are only locally energy minimizing or even unstable. In [24], we also proved that the interfaces of locally energy minimizing solutions as $\varepsilon \rightarrow 0$ converge to a locally area-minimizing hypersurface.

One motivation to consider (1.1) with variable $f$, aside from being a natural generalization, comes from the Cahn-Hilliard equation ([12]), which models various phase separation phenomena in a melted alloy with two stable phases. It is

$$
\begin{cases}u_{t}=\Delta f & \text { on } \Omega \times(0, \infty), \\ f=-\varepsilon \Delta u+\frac{W^{\prime}(u)}{\varepsilon}, & \\ \frac{\partial u}{\partial n}=\frac{\partial f}{\partial n}=0 & \text { on } \partial \Omega \times(0, \infty), \\ u(x, 0)=u_{0}(x) & \text { on } \Omega .\end{cases}
$$

The Neumann boundary condition reflects the insulation from the outside. It is a 4 -th order gradient flow of $E_{\varepsilon}(\cdot)$ with volume conservation:

$$
\frac{d}{d t} \int_{\Omega} u=0, \quad \frac{d}{d t} E_{\varepsilon}(u)=-\int_{\Omega}|\nabla f|^{2} \leq 0 .
$$

The time scale here is different from the usual setting, where $t$ may be replaced by $t \varepsilon$. For more physical background, derivation of the equation and the related equation such as the Allen-Cahn equation see for example, $[2,4,5,8,10,11,12,13,15,17,19,25]$ and the references therein. Even though it is far from a complete picture, we mention the rnost relevant references to the present article. With the timescale under consideration, it is known that the limit problem is the so-called Mullins-Sekerka problem ([32]). This was formally derived by Pego [34], and was given a rigorous justification using asymptotic expansions and spectral analysis by Alikakos, Bates and Chen [3]. In case of radial symmetry and Dirichlet conditions, Stoth [41] proved a global convergence to the limit problem in dimension three. For general domains and solutions, Chen [14] showed that the solutions converge to a weak solution of the limit problem using the notion of varifolds. Here, we take the similar approach to that of Chen in this paper, using varifold as our working device. 
PHASE FIELD MODEL

Given a sequence of solutions $\left\{u^{i}\right\}_{i=1}^{\infty}$ to

$$
-\varepsilon_{i} \Delta u^{i}+\frac{W^{\prime}\left(u^{i}\right)}{\varepsilon_{i}}=f^{i},
$$

$\varepsilon_{i} \rightarrow 0$, with uniform bounds $\sup _{i} E_{\varepsilon_{i}}\left(u^{i}\right) \leq E_{0}$ and $\sup _{i}\left\|f^{i}\right\|_{W^{1, n}}<\infty$, we show that there exists a subsequence whose interfaces converge in the Hausdorff distance sense to a hypersurface with the mean curvature determined by $f^{\infty}=\lim f^{i}, \sigma$ and the interface multiplicities. The mean curvature belongs to $L^{p}$ for any $p<\infty$ with respect to the $(n-1)$-dimensional hypersurface measure of the limit interface. We prove a monotonicity-type formula for the properly scaled energy, which extends the case discussed in [24]. Once this is established, the rectifiability and integrality of the limit varifold follow more or less from the argument in [24]. The proof is technically involved, and we need detailed estimates on the positive part of the so-called discrepancy function $\frac{\varepsilon}{2}|\nabla u|^{2}-\frac{W}{\varepsilon}$ using the Aleksandorov-Bakelman-Pucci (ABP) estimates. The results are applied to the Cahn-Hilliard equation with $n=2$, showing that there exists a subsequence for a.e. time with the convergence properties discussed in Theorem 1 when $\varepsilon \rightarrow 0$.

We note that there is an interesting class of unstable solutions with multiple peaks $([9,42,7,43]$ and the references therein). They describe neuclei of phase separation phenomena which are experimentally observed. Their singular limit cannot be captured by our method, since the chemical potential or energy has to blow-up in our scale.

The organization of the paper is as follows. In Section 2, we state our assumptions, terminologies and main results. In Section 3, we derive our main monotonicity formula for the scaled energy. In Section 4, we prove that the limit interface measure is supported on rectifiable sets and that the measure has a.e. integral densities. In the language of varifold, we show that the limit interface measure is an integral varifold (after dividing by $\sigma$ ) with the generalized mean curvature in $L^{p}$ for any $p<\infty$. In Section 5, we discuss some additional comments and some implications to the Cahn-Hilliard equation.

I would like to thank X.-F. Chen and Y. Giga for stimulating conversations in the course of the present research project.

\section{Assumptions AND MAIN RESUlts}

2.1. Hypotheses. We consider the problem with the following assumptions.

\section{Assumptions.}


A: The function $W: \mathbb{R} \rightarrow[0, \infty)$ is $C^{3}$ and $W( \pm 1)=0$. For some $\gamma \in(-1,1), W^{\prime}<0$ on $(\gamma, 1)$ and $W^{\prime}>0$ on $(-1, \gamma)$. For some $\alpha \in(0,1)$ and $\kappa>0, W^{\prime \prime}(x) \geq \kappa$ for all $|x| \geq \alpha$.

B: $U \subset \mathbb{R}^{n}$ is a bounded open set with Lipschitz boundary $\partial U$. Sequences of $C^{3}(U)$ functions $\left\{u^{i}\right\}_{i=1}^{\infty}$ and $C^{1}(U)$ functions $\left\{f^{i}\right\}_{i=1}^{\infty}$ satisfy

$$
\varepsilon_{i} \Delta u^{i}=\varepsilon_{i}^{-1} W^{\prime}\left(u^{i}\right)-f^{i}
$$

on $U$. Here, $\lim _{i \rightarrow \infty} \varepsilon_{i}=0$, and we assume there exist

$c_{0}, \lambda_{0}$ and $E_{0}$ such that $\sup _{U}\left|u^{i}\right| \leq c_{0},\left\|f^{i}\right\|_{W^{1, n}(U)}=\left(\int_{U}\left|f^{i}\right|^{n}+\right.$ $\left.\left|\nabla f^{i}\right|^{n}\right)^{1 / n} \leq \lambda_{0}$ and

$$
\int_{U} \frac{\varepsilon_{i}\left|\nabla u^{i}\right|^{2}}{2}+\frac{W\left(u^{i}\right)}{\varepsilon_{i}} \leq E_{0}
$$

for all $i$.

Assumption A requires that $W$ has a standard W-shape with nondegenerate minima at \pm 1 , and local maximum at $\gamma$. The assumptions on $u^{i}$ and $f^{i}$ are either motivated by the applications to the CahnHilliard equation (see Section 5.2) or regarded simply as a starting point of the problem. The regularity of $u$ is then standard ([21]). The requirement of $W^{1, n}$ norm bound on $f^{i}$ comes mainly from a technical limit of our approach using the ABP estimates. It is not clear this can be replaced by a weaker norm bound such as $W^{1, p}$ for some $p>n / 2$, which we believe is ultimately the weakest bound for $f^{i}$ to conclude the similar convergence results (see Section 5.1 for more discussion). Let

We next discuss a few immediate consequences of the assumptions.

$$
\Phi(s)=\int_{0}^{s} \sqrt{W(s) / 2} d s
$$

and define new functions

$$
w^{i}=\Phi \circ u^{i}
$$

for each $i$.

Since $\left|\nabla w^{i}\right|=\sqrt{W\left(u^{i}\right) / 2}\left|\nabla u^{i}\right|$, it follows by the Cauchy-Schwartz inequality that

$$
\int_{U}\left|\nabla w^{i}\right| \leq \frac{1}{2} \int_{U} \frac{\varepsilon_{i}\left|\nabla u^{i}\right|^{2}}{2}+\frac{W\left(u^{i}\right)}{\varepsilon_{i}} \leq \frac{E_{0}}{2} .
$$

We also have $\Phi\left(-c_{0}\right) \leq w^{i} \leq \Phi\left(c_{0}\right)$. By the compactness theorem for bounded variation functions, there exists a subsequence also denoted 
PHASE FIELD MODEL

by $\left\{w^{i}\right\}$ and an a.e. pointwise limit $w^{\infty}$, such that

$$
\lim _{i \rightarrow \infty} \int_{U}\left|w^{i}-w^{\infty}\right|=0 \text { and } \int_{U}\left|D w^{\infty}\right| \leq \liminf _{i \rightarrow \infty} \int_{U}\left|\nabla w^{i}\right| .
$$

Here, $\left|D w^{\infty}\right|$ is the total variation of the vector-valued Radon measure $D w^{\infty}$.

Let $\Phi^{-1}$ be the inverse of $\Phi$ and define

$$
u^{\infty}=\Phi^{-1}\left(w^{\infty}\right)
$$

Then $u^{i} \rightarrow u^{\infty}$ a.e., and by the Lebesgue dominated convergence theorem

$$
\int_{U}\left|u^{i}-u^{\infty}\right| \rightarrow 0
$$

Also by Fatou's Lemma and the energy bound, we have

$$
\int_{U} W\left(u^{\infty}\right)=\int_{U} \lim _{i \rightarrow \infty} W\left(u^{i}\right) \leq \liminf _{i \rightarrow \infty} \int_{U} W\left(u^{i}\right)=0 .
$$

This shows that $u^{\infty}= \pm 1$ a.e. on $U$, and the sets $\left\{u^{\infty}= \pm 1\right\}$ have finite perimeter in $U$, since

$$
\left\|\partial\left\{u^{\infty}=1\right\}\right\|(U)=\frac{1}{2} \int_{U}\left|D u^{\infty}\right|=\frac{1}{\sigma} \int_{U}\left|D w^{\infty}\right| \leq \frac{E_{0}}{2 \sigma},
$$

where we define

$$
\sigma=\int_{-1}^{1} \sqrt{W(s) / 2} d s
$$

and where $\|\partial A\|$ denotes the perimeter of $A$ in the measure-theoretic sense (see [18]).

2.2. The associated varifolds. In this section we associate to each solution of (2.1) a varifold in a natural way. We refer to $[1,38]$ for a comprehensive treatment of varifolds.

Let $G(n, n-1)$ denote the Grassman manifold of unoriented $(n-1)$ dimensional planes in $\mathbb{R}^{n}$. We say $V$ is an $(n-1)$-dimensional varifold in $U \subset \mathbb{R}^{n}$ if $V$ is a Radon measure on $G_{n-1}(U)=U \times G(n, n-1)$. Let $V_{n-1}(U)$ denote the set of all $(n-1)$-dimensional varifolds in $U$. Convergence in the varifold sense means convergence in the usual sense of measures. For $V \in V_{n-1}(U)$, we let the weight $\|V\|$ be the Radon measure in $U$ defined by

$$
\|V\|(A)=V(\{(x, S) \mid x \in A, S \in G(n, n-1)\})
$$

for each Borel set $A \subset U$. If $M$ is a $(n-1)$-rectifiable subset of $U$ we define $v(M) \in V_{n-1}(U)$ by

$$
v(M)(E)=\mathcal{H}^{n-1}\left(\left\{x \in U \mid\left(x, \operatorname{Tan}^{n-1}\left(\mathcal{H}^{n-1}\left\lfloor_{M}, x\right)\right) \in E\right\}\right)\right.
$$




\section{YOSHIHIRO TONEGAWA}

for each Borel set $E \in G_{n-1}(U)$, where $\operatorname{Tan}^{n-1}\left(\mathcal{H}^{n-1}\left\lfloor_{M}, x\right)\right.$ is the approximate tangent plane to $M$ at $x$ and so exists for $\mathcal{H}^{n-1}$ a.e. $x \in M$.

We associate to each function $w^{i}$ a varifold $V^{i}$ defined naturally as follows $([14,24,25])$. By Sard's theorem, $\left\{w^{i}=t\right\} \subset U$ is a $C^{3}$ hypersurface for $L^{1}$ almost all $t$. Define $V^{i} \in V_{n-1}(U)$ by

$$
V^{i}(A)=\int_{-\infty}^{\infty} v\left(\left\{w^{i}=t\right\}\right)(A) d t
$$

for each Borel set $A \subset G_{n-1}(U)$. By the coarea formula ([18]), we have

$$
\left\|V^{i}\right\|(A)=\int_{-\infty}^{\infty} \mathcal{H}^{n-1}\left(\left\{w^{i}=t\right\} \cap A\right) d t=\int_{A}\left|\nabla w^{i}\right|
$$

for each Borel set $A \subset U$. One may interpret the varifold $V^{i}$ as a weighted averaging of the level sets of $u^{i}$, which is concentrated around the transition region. The first variation of $V^{i}$ is given by (see [33, Section 2.1])

$$
\delta V^{i}(g)=\int_{U}\left(\operatorname{div} g-\sum_{j, k=1}^{n} \frac{w_{x_{j}}^{i}}{\left|\nabla w^{i}\right|} \frac{w_{x_{k}}^{i}}{\left|\nabla w^{i}\right|} g_{x_{k}}^{j}\right)\left|\nabla w^{i}\right|
$$

for each $g \in C_{c}^{1}\left(U ; \mathbb{R}^{n}\right)$.

2.3. Main results. With the above terminology and assumptions $\mathbf{A}$ and $\mathbf{B}$, we show the following.

Theorem 1. Let $V^{i}$ be the varifold associated with $u^{i}$ (via $\left.w^{i}\right)$ as in Sections 2.1 and 2.2. On passing to a subsequence we can assume $f^{i} \rightarrow f^{\infty}$ weakly in $W^{1, n}, \quad u^{i} \rightarrow u^{\infty}$ a.e., $\quad V^{i} \rightarrow V$ in the varifold sense.

Moreover,

(1) For each $\phi \in C_{c}(U)$,

$$
\|V\|(\phi)=\lim _{i \rightarrow \infty} \int \phi \frac{\varepsilon_{i}\left|\nabla u^{i}\right|^{2}}{2}=\lim _{i \rightarrow \infty} \int \phi \frac{W\left(u^{i}\right)}{\varepsilon_{i}}=\lim _{i \rightarrow \infty} \int \phi\left|\nabla w^{i}\right| .
$$

(2) $\operatorname{supp}\left\|\partial\left\{u^{\infty}=1\right\}\right\| \subset \operatorname{supp}\|V\|$, and $\left\{u^{i}\right\}$ converges locally uniformly to \pm 1 on $U \backslash \operatorname{supp}\|V\|$.

(3) For each $\tilde{U} \subset \subset U$ and $0<b<1,\left\{\left|u^{i}\right| \leq 1-b\right\} \cap \tilde{U}$ converges to $\tilde{U} \cap \operatorname{supp}\|V\|$ in the Hausdorff distance sense.

(4) $\sigma^{-1} V$ is an integral varifold. Moreover, the density $\theta(x)=\sigma N(x)$ of $V$ satisfies

$$
N(x)=\left\{\begin{array}{lll}
\text { odd } & \mathcal{H}^{n-1} & \text { a.e. } x \in M^{\infty} \\
\text { even } & \mathcal{H}^{n-1} & \text { a.e. } x \in \operatorname{supp}\|V\| \backslash M^{\infty}
\end{array}\right.
$$

where $M^{\infty}$ is the reduced boundary of $\left\{u^{\infty}=1\right\}$. 
PHASE FIELD MODEL

(5) The generalized mean curvature $H$ of $V$ is given by

$$
H(x)= \begin{cases}\frac{f^{\infty}(x)}{\theta(x)} \nu^{\infty}(x) & \mathcal{H}^{n-1} \text { a.e. } x \in M^{\infty}, \\ 0 & \mathcal{H}^{n-1} \text { a.e. } x \in \operatorname{supp}\|V\| \backslash M^{\infty},\end{cases}
$$

where $\nu^{\infty}$ is the inward normal for $M^{\infty}$.

(6) The generalized mean curvature $H$ belongs to $L_{\text {loc }}^{p}$ for any $p<\infty$ with respect to $\|V\|$.

Comments which follows Theorem 1 in [24] goes with minor changes here as well. Part (1) shows that in the limit the energy is equally divided between the two terms of the energy functional (1.2), called the equipartition of energy. In fact, our result shows the following:

For any $\tilde{U} \subset \subset U$ and for the full sequence (not only a subsequence),

$$
\left.\lim _{i \rightarrow \infty} \int_{\tilde{U}}\left|\frac{\varepsilon_{i}}{2}\right| \nabla u^{i}\right|^{2}-\frac{W\left(u^{i}\right)}{\varepsilon_{i}} \mid=0 .
$$

This is even without $\frac{\varepsilon_{i}}{2}\left|\nabla u^{i}\right|^{2} d x$ converging to some measure. Part (4) suggests that folding of the interface as $\varepsilon \rightarrow 0$ occurs locally as an integer multiple of 1-D traveling wave solutions ([29]), almost everywhere in the measure-theoretic sense.

Without loss of generality, we may assume that $M^{\infty} \subset \operatorname{supp} \| \partial\left\{u^{\infty}=\right.$ $1\} \|$. We were not able to prove or disprove that $\mathcal{H}^{n-1}\left(\operatorname{supp} \| \partial\left\{u^{\infty}=\right.\right.$ $\left.1\} \| \backslash M^{\infty}\right)=0$ in general. This is due to the lack of a uniform lower density estimate for the measure $\left\|\partial\left\{u^{\infty}=1\right\}\right\|$ (as opposed to $\|V\|$ ) at $\mathcal{H}^{n-1}$ a.e. $x$ in the closure of $M^{\infty}$. On the other hand, if $N(x)$ is odd $\mathcal{H}^{n-1}$ a.e. for $x \in \operatorname{supp}\|V\|$, the result (4) shows that $\mathcal{H}^{n-1}(\operatorname{supp}\|V\| \backslash$ $\left.M^{\infty}\right)=0$ and $\operatorname{supp}\|V\|=\operatorname{supp}\left\|\partial\left\{u^{\infty}=1\right\}\right\|$. If $N(x)=1$ a.e., then this corresponds to "no energy loss" situation, since

$$
\int\left|D w^{\infty}\right| \phi=\sigma\left\|\partial\left\{u^{\infty}=1\right\}\right\|(\phi)=\|V\|(\phi)=\lim _{i \rightarrow \infty} \int\left|\nabla w^{i}\right| \phi
$$

for all $\phi \in C_{c}(U)$. In case $f^{i}$ are all constants and with no energy loss, the relation between the curvature of the limit interface and the chemical potential is established by Luckhaus and Modica in [27], and we prove here the direct generalization.

It is well-known that the support of a rectifiable varifold with $L^{p}$ mean curvature, $p>n-1$, is locally a $C^{1, \alpha}$ graph on a relatively open dense subset $\mathcal{O}([1])$ for $\alpha=1-\frac{n-1}{p}$. The density function on $\mathcal{O}$ is locally constant and integer valued. If we additionally assume a better bound on $f^{i}$, for example, $C^{k, \alpha}, 0<\alpha<1, k \geq 0$, standard elliptic estimates [21] show that the support there is $C^{k+2, \alpha}$. On the other hand, we do not know if $\mathcal{H}^{n-1}(\operatorname{supp}\|V\| \backslash \mathcal{O})=0$ in general. The 


\section{YOSHIHIRO TONEGAWA}

density function $\theta$ is defined everywhere on the support of $\|V\|$ and is upper-semicontinuous.

If $N=1, \mathcal{H}^{n-1}$ a.e. on supp $\|V\|$, then the support is locally a $C^{1, \alpha}$ hypersuface of mean curvature given by $f^{\infty} / \sigma$, except for a closed set of $\mathcal{H}^{n-1}$ measure zero.

\section{MONOTONICITY FORMULA}

In this section, in addition to Assumption $\mathbf{A}$, we assume that the functions $u, f: U \rightarrow \mathbb{R}$ satisfy Assumption $\mathbf{B}$ with $u^{i}, f^{i}$ and $\varepsilon_{i}$ there replaced by $u, f$ and $\varepsilon$ respectively. We assume that $\widetilde{U}$ is open and $\widetilde{U} \subset \subset U$.

The main result here is the energy monotonicity formula for

$$
E(r, x)=\frac{1}{r^{n-1}} \int_{B_{r}(x)}\left(\frac{\varepsilon|\nabla u|^{2}}{2}+\frac{W(u)}{\varepsilon}\right),
$$

Proposition 3.6. We first derive the identity (3.1) which gives the expression of the radial derivative of $E(r, x)$. The main difficulty to prove the positivity of $\frac{d}{d r} E(\dot{r}, x)$ comes from the positive part of the discrepancy function $\xi_{\varepsilon}=\frac{\varepsilon|\nabla u|^{2}}{2}-\frac{W(u)}{\varepsilon}$. When $f$ is a constant function, we proved in [24] that $\xi_{\varepsilon} \leq c$ independent of $\varepsilon$. There we utilized a differential inequality satisfied by $\xi_{\varepsilon}-G(u)$, where $G$ is a suitable modification function, and the maximum principle type argument . In this paper, since we only have control of $\nabla f$ in $L^{n}$-norm, we use the Aleksandorov-Bakelman-Pucci type estimate instead. Even though we could not prove the uniform supremum bound, we show $\xi_{\varepsilon} \leq c \varepsilon^{-2 / 5}$ for all sufficiently small $\varepsilon$ (Proposition 3.2). This estimate gives us an energy lower bound around the interface for the length scale of order $\varepsilon^{2 / 5}$. Then we use this lower bound and a covering lemma to obtain the estimate on the integral of $\xi_{\varepsilon}$ (Lemma 3.5) satisfied for $r$ larger than the length scale of order $\varepsilon^{2 / 5}$. Combined with this estimate, we obtain the monotonicity formula (3.10).

We denote $W-\varepsilon u f$ by $\widetilde{W}$.

Lemma 3.1. For $B_{r}(x) \subset \subset U$, we have

$$
\begin{gathered}
\frac{d}{d r}\left\{\frac{1}{r^{n-1}} \int_{B_{r}(x)}\left(\frac{\varepsilon|\nabla u|^{2}}{2}+\frac{\widetilde{W}}{\varepsilon}\right)\right\}=\frac{1}{r^{n}} \int_{B_{r}(x)}\left(\frac{\widetilde{W}}{\varepsilon}-\frac{\varepsilon|\nabla u|^{2}}{2}\right) \\
+\frac{\varepsilon}{r^{n+1}} \int_{\partial_{B_{r}(x)}}((y-x) \cdot \nabla u)^{2}-\frac{1}{r^{n}} \int_{B_{r}(x)}((y-x) \cdot \nabla f) u
\end{gathered}
$$


Proof. Multiply both sides of $(1.2)$ by $\nabla u \cdot g$, where $g=\left(g^{1}, \cdots, g^{n}\right) \in$ $C_{c}^{1}\left(U ; \mathbb{R}^{n}\right)$. Then, after two integrations by parts, we obtain

$$
\int_{U}\left(\left(\frac{\varepsilon|\nabla u|^{2}}{2}+\frac{\widetilde{W}(u)}{\varepsilon}\right) \operatorname{div} g-\varepsilon \sum_{i, j} u_{y_{i}} u_{y_{j}} g_{y_{i}}^{j}-(\nabla f \cdot g) u\right)=0
$$

We let $x=0$ by a suitable translation and let $g^{j}(y)=y_{j} \rho(|y|)$, where $\rho(|y|)$ is a smooth approximation to the characteristic function $\chi_{B_{r}}$. Writing $r=|y|,(3.2)$ becomes

$$
\begin{gathered}
\int_{U}\left(\left(\frac{\varepsilon|\nabla u|^{2}}{2}+\frac{\widetilde{W}(u)}{\varepsilon}\right)\left(r \rho^{\prime}+n \rho\right)-\varepsilon \frac{\rho^{\prime}}{r}(y \cdot \nabla u)^{2}-\varepsilon|\nabla u|^{2} \rho\right. \\
-(\nabla f \cdot g) \rho u)=0 .
\end{gathered}
$$

By letting $\rho \rightarrow \chi_{B_{r}}$ and rearranging terms, we obtain

$$
\begin{aligned}
-(n-1) & \int_{B_{r}}\left(\frac{\varepsilon|\nabla u|^{2}}{2}+\frac{\widetilde{W}(u)}{\varepsilon}\right)+r \int_{\partial B_{r}}\left(\frac{\varepsilon|\nabla u|^{2}}{2}+\frac{\widetilde{W}(u)}{\varepsilon}\right) \\
& =\int_{B_{r}}\left(\frac{\widetilde{W}(u)}{\varepsilon}-\frac{\varepsilon|\nabla u|^{2}}{2}\right)+\frac{\varepsilon}{r} \int_{\partial B_{r}}(y \cdot \nabla u)^{2}-\int_{B_{r}}(y \cdot \nabla f) u .
\end{aligned}
$$

By dividing the above expression by $r^{n}$, we obtain (3.1).

For the moment we assume the following technical result which we prove later.

Proposition 3.2. There exist constants $c_{1}$ and $\varepsilon_{1}$ which depend only on $c_{0}, \lambda_{0}, W, n$ and $\operatorname{dist}(\widetilde{U}, \partial U)$ such that, if $\varepsilon<\varepsilon_{1}$,

$$
\sup _{\widetilde{U}}\left(\frac{\varepsilon|\nabla u|^{2}}{2}-\frac{W}{\varepsilon}\right) \leq c_{1} \varepsilon^{-2 / 5} \text {. }
$$

Lemma 3.3. For $B_{r}(x) \subset \widetilde{U}$, there exists $c_{2}$ which depends only on $c_{0}, \lambda_{0}, n, W$ and $\operatorname{dist}(\widetilde{U}, \partial U)$ such that, for any $s<r$ and $\varepsilon<\varepsilon_{1}$,

$$
\begin{gathered}
E(r, x)-E(s, x) \geq-c_{2}\left(r+r^{1 / 2}+r \varepsilon^{-2 / 5}\right) \\
+\int_{s}^{r} \frac{d \tau}{\tau^{n}} \int_{B_{\tau}(x)}\left(\frac{W}{\varepsilon}-\frac{\varepsilon|\nabla u|^{2}}{2}\right)^{+} .
\end{gathered}
$$

Proof. By (3.3), the Hölder and Sobolev inequalities, we obtain

$$
\begin{aligned}
& \frac{1}{r^{n}} \int_{B_{r}(x)}\left(\frac{\varepsilon|\nabla u|^{2}}{2}-\frac{\widetilde{W}}{\varepsilon}\right)^{+} \leq \frac{1}{r^{n}} \int_{B_{r}(x)}\left\{\left(\frac{\varepsilon|\nabla u|^{2}}{2}-\frac{W}{\varepsilon}\right)^{+}+|u f|\right\} \\
& \leq \omega_{n}\left(c_{1} \varepsilon^{-2 / 5}+c_{0}\|f\|_{L^{2 n} r^{-1 / 2}}\right) \leq \omega_{n}\left(c_{1} \varepsilon^{-2 / 5}+c_{0} c(n) \lambda_{0} r^{-1 / 2}\right) .
\end{aligned}
$$




\section{YOSHIHIRO TONEGAWA}

After integrating over $[s, r]$, this gives the bound on the negative part of the first term in (3.1). Also, for the third term in (3.1),

$$
\begin{aligned}
\left|\frac{1}{r^{n}} \int_{B_{r}(x)}((y-x) \cdot \nabla f) u\right| & \leq c_{0} \frac{1}{r^{n-1}} \int_{B_{r}(x)}|\nabla f| \\
\leq c_{0} c(n)\|\nabla f\|_{L^{n}} & \leq c_{0} c(n) \lambda_{0} .
\end{aligned}
$$

The difference resulting from $W$ and $\widetilde{W}$ may be estimated by

$$
\frac{1}{r^{n-1}} \int_{B_{r}(x)}|u f| \leq c_{0} c(n)\|f\|_{W^{1, n}} r^{1 / 2} \leq c_{0} c(n) \lambda_{0} r^{1 / 2}
$$

With an appropriate choice of $c_{2}$, we obtain (3.4).

Proposition 3.4. There exist constants $0<c_{3}, c_{4}<1$ which depend only on $c_{0}, \lambda_{0}, n, W$ and $\operatorname{dist}(\widetilde{U}, \partial U)$ such that, if $B_{\varepsilon^{2 / 5}}(x) \subset \widetilde{U}$, $|u(x)| \leq \alpha$ and $\varepsilon<\varepsilon_{1}$, then

$$
E(r, x) \geq c_{4} \quad \text { for } \varepsilon \leq r \leq c_{3} \varepsilon^{2 / 5} .
$$

Proof. Translate $x$ to the origin. By scaling $\tilde{x}=x / \varepsilon$ and $\tilde{u}(\tilde{x})=u(\varepsilon \tilde{x})$, the energy scales as

$$
E(\varepsilon, 0)=\frac{1}{\varepsilon^{n-1}} \int_{B_{\varepsilon}}\left(\frac{\varepsilon|\nabla u|^{2}}{2}+\frac{W}{\varepsilon}\right) d x=\int_{B_{1}}\left(\frac{|\nabla \tilde{u}|^{2}}{2}+W\right) d \tilde{x} .
$$

In this scale, there exists a constant $c_{5}$ which depends only on $c_{0}, \lambda_{0}, n$, $W$ and $\operatorname{dist}(\widetilde{U}, \partial U)$ such that $|\nabla \tilde{u}| \leq c_{5}$ due to the standard elliptic $L^{p}$ estimate ([21]). Since $|\tilde{u}(0)| \leq \alpha$, there exists some $c_{6}=c\left(c_{5}, W\right)>0$ such that $W(\tilde{u}) \geq c_{6}$ on $B_{c_{6}}$. Hence, $E(\varepsilon, 0) \geq \omega_{n} c_{6}^{n+1}$. Using (3.4) and setting $s=\varepsilon$, if we restrict $r$ to be less than $c_{3} \varepsilon^{2 / 5}$ for sufficiently small $c_{3}=c\left(c_{2}, c_{6}, n\right)$ and setting $c_{4}=\omega_{n} c_{6}^{n+1} / 2$, we obtain the desired inequality (3.5).

In the following, we use the energy lower bound (3.5) around the interface. The ball $B_{r}(x)$ we consider has a radius of order $\varepsilon^{2 / 5}$ at least, which is larger than $\varepsilon^{4 / 5}$, where we have the energy lower bound. We cover the interface by a collection of balls with the radius $\varepsilon^{4 / 5}$.

Proposition 3.5. There exist constants $c_{7}, c_{8}, \varepsilon_{2} \leq \varepsilon_{1}$ which depend only on $c_{0}, \lambda_{0}, n, W$ and $\operatorname{dist}(\widetilde{U}, \partial U)$ such that, if $B_{r}(x) \subset \widetilde{U}, r \geq$ $c_{3} \varepsilon^{2 / 5}$ and $\varepsilon<\varepsilon_{2}$,

$$
\frac{1}{r^{n}} \int_{B_{r}(x)}\left(\frac{\varepsilon|\nabla u|^{2}}{2}-\frac{W}{\varepsilon}\right)^{+} \leq c_{7} E(r, x)+c_{8}
$$


Proof. We translate $x$ to the origin. We estimate the integral on

$$
\begin{aligned}
& \mathcal{A}=\left\{x \in B_{r} \backslash B_{r-\varepsilon^{4 / 5}}\right\} \\
& \mathcal{B}=\left\{x \in B_{r-\varepsilon^{4 / 5}} \mid \operatorname{dist}(\{|u| \leq \alpha\}, x)<\varepsilon^{4 / 5}\right\} \\
& \mathcal{C}=\left\{x \in B_{r-\varepsilon^{4 / 5}} \mid \operatorname{dist}(\{|u| \leq \alpha\}, x) \geq \varepsilon^{4 / 5}\right\}
\end{aligned}
$$

The set $\mathcal{A}$ is the $\varepsilon^{4 / 5}$-shell of the ball $B_{r}, \mathcal{B}$ is the $\varepsilon^{4 / 5}$-neighborhood of the interface and $\mathcal{C}$ is the complement of the two.

(1) Estimate on $\mathcal{A}$. By (3.3),

$$
\frac{1}{r^{n}} \int_{\mathcal{A}}\left(\frac{\varepsilon|\nabla u|^{2}}{2}-\frac{W}{\varepsilon}\right)^{+} \leq \frac{n \omega_{n} \varepsilon^{4 / 5}}{r} c_{1} \varepsilon^{-2 / 5} \leq \frac{n \omega_{n} c_{1}}{c_{3}}
$$

since $r \geq c_{3} \varepsilon^{2 / 5}$.

(2) Estimate on $\mathcal{B}$. We first estimate $\mathcal{H}^{n}(\mathcal{B})$. We apply the Vitali covering lemma ([38, Theorem 3.3]) to the family of balls $\left\{B_{\varepsilon^{4 / 5}}(x)\right\}_{x \in\{|u| \leq \alpha\} \cap \mathcal{B}}$ (which covers $\mathcal{B}$ ), so that $\left\{B_{\varepsilon^{4 / 5}}\left(x_{i}\right)\right\}_{i=1}^{N}$ is a pairwise disjoint subset of the family and so that $\mathcal{B} \subset \cup_{i=1}^{N} B_{5 \varepsilon^{4 / 5}}\left(x_{i}\right)$. Then we have $\mathcal{H}^{n}(\mathcal{B}) \leq$ $\omega_{n}\left(5 \varepsilon^{4 / 5}\right)^{n} N$. On the other hand, by (3.5) and since $\varepsilon<\varepsilon^{4 / 5}<c_{3} \varepsilon^{2 / 5}$ for all sufficiently small $\varepsilon$,

$$
c_{4}\left(\varepsilon^{4 / 5}\right)^{n-1} \leq \int_{B_{\varepsilon^{4 / 5}\left(x_{i}\right)}}\left(\frac{\varepsilon|\nabla u|^{2}}{2}+\frac{W}{\varepsilon}\right)
$$

holds for each $i=1, \cdots, N$. Since they are pairwise disjoint, by summing over $i$, we have

$$
N c_{4}\left(\varepsilon^{4 / 5}\right)^{n-1} \leq \int_{B_{r}}\left(\frac{\varepsilon|\nabla u|^{2}}{2}+\frac{W}{\varepsilon}\right)
$$

These two estimates show that

$$
\mathcal{H}^{n}(\mathcal{B}) \leq \frac{\omega_{n} 5^{n} \varepsilon^{4 / 5}}{c_{4}} \int_{B_{r}}\left(\frac{\varepsilon|\nabla u|^{2}}{2}+\frac{W}{\varepsilon}\right)=\frac{\omega_{n} 5^{n} \varepsilon^{4 / 5} r^{n-1} E(r, 0)}{c_{4}}
$$

Finally, with (3.3),

$$
\frac{1}{r^{n}} \int_{\mathcal{B}}\left(\frac{\varepsilon|\nabla u|^{2}}{2}-\frac{W}{\varepsilon}\right)^{+} \leq \frac{c_{1} \varepsilon^{-2 / 5} \mathcal{H}^{n}(\mathcal{B})}{r^{n}} \leq \frac{\omega_{n} 5^{n} c_{1} E(r, 0)}{c_{3} c_{4}}
$$

since $r \geq c_{3} \varepsilon^{2 / 5}$.

(3) Estimate on $\mathcal{C}$. Define a Lipschitz function $\phi$ as follows:

$$
\phi(x)=\min \left\{1, \varepsilon^{-4 / 5} \operatorname{dist}(\{|x| \geq r\} \cup\{|u| \leq \alpha\}, x)\right\} .
$$

$\phi$ is 0 on the set $\{|u| \leq \alpha\} \cup\{|x| \geq r\}, 1$ on $\mathcal{C}$, and $|\nabla \phi| \leq \varepsilon^{-4 / 5}$. Using this $\phi$, we estimate $\frac{\varepsilon|\nabla u|^{2}}{2}$ (which is larger than $\left(\frac{\varepsilon|\nabla u|^{2}}{2}-\frac{W}{\varepsilon}\right)^{+}$) 


\section{YOSHIHIRO TONEGAWA}

on $\mathcal{C}$. Differentiate the equation (1.2) with respect to the $k$-th variable, multiply it by $u_{y_{k}} \phi^{2}$ and sum over $k$. Then,

$$
\int \sum_{k=1}^{n} \varepsilon u_{y_{k}} \Delta u_{y_{k}} \phi^{2}=\int \frac{W^{\prime \prime}}{\varepsilon}|\nabla u|^{2} \phi^{2}-\nabla f \cdot \nabla u \phi^{2} .
$$

We integrate by parts, and since $W^{\prime \prime} \geq \kappa$ on $\{|u| \geq \alpha\}$,

$$
\int \frac{\kappa|\nabla u|^{2} \phi^{2}}{\varepsilon}+\varepsilon\left|\nabla^{2} u\right|^{2} \phi^{2} \leq \int|\nabla f||\nabla u| \phi^{2}+2 \varepsilon \sum_{k, l=1}^{n}\left|\phi u_{y_{k}} \phi_{y_{l}} u_{y_{k} y_{l}}\right| .
$$

By the Cauchy-Schwartz inequality, we then obtain

$$
(L H S) \leq \int \frac{\varepsilon}{2 \kappa}|\nabla f|^{2} \phi^{2}+\frac{\kappa}{2 \varepsilon}|\nabla u|^{2} \phi^{2}+\frac{\varepsilon}{2} \phi^{2}\left|\nabla^{2} u\right|^{2}+2 \varepsilon|\nabla \phi|^{2}|\nabla u|^{2} .
$$

Relegating two terms to the (LHS), we have

$$
\frac{\kappa}{2 \varepsilon} \int|\nabla u|^{2} \phi^{2} \leq \int \frac{\varepsilon}{2 \kappa}|\nabla f|^{2} \phi^{2}+2 \varepsilon|\nabla \phi|^{2}|\nabla u|^{2} .
$$

Since $|\nabla \phi| \leq \varepsilon^{-4 / 5}$

$$
\int_{\mathcal{C}} \varepsilon|\nabla u|^{2} \leq \int_{B_{r}} \frac{\varepsilon^{3}|\nabla f|^{2}}{\kappa^{2}}+\frac{4 \varepsilon^{7 / 5}|\nabla u|^{2}}{\kappa}
$$

By the Hölder inequality,

$$
\begin{aligned}
\frac{1}{r^{n}} \int_{\mathcal{C}} \frac{\varepsilon|\nabla u|^{2}}{2} & \leq \frac{\varepsilon^{3}\left(\omega_{n} r^{n}\right)^{\frac{n-2}{n}}|| \nabla f \|_{L^{2}}^{2}}{2 \kappa^{2} r^{n}}+\frac{2 \varepsilon^{2 / 5} E(r, 0)}{\kappa r} \\
& \leq \frac{\lambda_{0}^{2} \omega_{n}^{\frac{n-2}{n}} \varepsilon^{11 / 5}}{2 \kappa^{2} c_{3}^{2}}+\frac{2 E(r, 0)}{\kappa c_{3}}
\end{aligned}
$$

by $r \geq c_{3} \varepsilon^{2 / 5}$. With appropriate choices of the constants, we obtain (3.6) by $(3.7-9)$.

Proposition 3.6. There exist constants $c_{9}, r_{0}$ depending only on $c_{0}$, $\lambda_{0}, n, W$ and $\operatorname{dist}(\widetilde{U}, \partial U)$, with the following property: For any $B_{r}(x) \subset$ $\widetilde{U}, \varepsilon<\varepsilon_{2}$ and $c_{3} \varepsilon^{2 / 5} \leq s<r \leq r_{0}$,

$$
e^{c_{7} r} E(r, x)-e^{c_{7} s} E(s, x) \geq \int_{s}^{r} \frac{e^{c_{7} \tau}}{\tau^{n}} \int_{B_{\tau}}\left(\frac{W}{\varepsilon}-\frac{\varepsilon|\nabla u|^{2}}{2}\right)^{+} d \tau-c_{9} r^{1 / 2} .
$$

Proof. By (3.1) and (3.6), we obtain

$$
\frac{d}{d r} E(r, x)+\frac{d}{d r}\left(\frac{1}{r^{n}} \int_{B_{r}(x)} u f\right) \geq \frac{1}{r^{n}} \int_{B_{r}(x)}\left(\frac{W}{\varepsilon}-\frac{\varepsilon|\nabla u|^{2}}{2}\right)^{+}
$$


PHASE FIELD MODEL

$$
-c_{7} E(r, x)-c_{8}-\frac{1}{r^{n}} \int_{B_{r}(x)}(|u f|+r|\nabla f||u|) .
$$

The last term is bounded from below by $-c_{10}\left(n, c_{0}, \lambda_{0}\right) r^{-1 / 2}$. By multiplying $e^{c_{7} r}$ to the both sides and integrating over $[s, r]$,

$$
\begin{gathered}
e^{c_{7} r} E(r, x)-e^{c_{7} s} E(s, x) \geq \int_{s}^{r} e^{c_{7} \tau} \frac{d}{d \tau}\left(\frac{1}{\tau^{n-1}} \int_{B_{\tau}(x)} u f\right) d \tau \\
+\int_{s}^{r} \frac{e^{c_{7} \tau}}{\tau^{n}} \int_{B_{\tau}}\left(\frac{W}{\varepsilon}-\frac{\varepsilon|\nabla u|^{2}}{2}\right)^{+} d \tau-2 c_{10} r^{1 / 2} e^{c_{7} r}-c_{8} c_{7}^{-1}\left(e^{c_{7} r}-e^{c_{7} s}\right) .
\end{gathered}
$$

The first term in the (RHS) is estimated from below by $-c\left(r^{1 / 2}+r^{3 / 2}\right)$, thus with a suitable restriction on $r$, we obtain (3.10).

For the rest of the section, we show Proposition 3.2. First, we need the following lemma showing that $|u|$ stays within $1+\varepsilon^{\eta}$ for $\eta<1$. In case $f$ is bounded in $L^{\infty}$-norm, we can use the pointwise maximum principle as in [24, Proposition 3.2]. Here we need to use integral estimates.

Proposition 3.7. There exist constants $\varepsilon_{3}$ which depend only on $\lambda_{0}$, $c_{0}, n, 0<\eta<1, \operatorname{dist}(\widetilde{U}, \partial U)$ and $W$ such that

$$
\sup _{\widetilde{U}}|u| \leq 1+\varepsilon^{\eta}
$$

whenever $\varepsilon \leq \varepsilon_{3}$.

Proof. Suppose $B_{1} \subset \widetilde{U}$. For any $p \geq 1$, multiply both sides of the equation (1.2) by $\left[(u-1)^{+}\right]^{p} \phi^{2}$, where $\phi \in C_{c}^{\infty}\left(B_{1}\right), \phi \geq 0$, and integrate by parts. Then we obtain

$$
\begin{gathered}
-\varepsilon \int p\left[(u-1)^{+}\right]^{p-1}|\nabla u|^{2} \phi^{2}+\left[(u-1)^{+}\right]^{p} 2 \phi \nabla \phi \cdot \nabla u \\
=\int \frac{W^{\prime}}{\varepsilon}\left[(u-1)^{+}\right]^{p} \phi^{2}-\int f\left[(u-1)^{+}\right]^{p} \phi^{2} .
\end{gathered}
$$

For $u \geq 1, W^{\prime}(u) \geq \kappa(u-1)$ by the Assumption $\mathbf{B}$, hence

$$
\begin{gathered}
\frac{\kappa}{\varepsilon} \int\left[(u-1)^{+}\right]^{p+1} \phi^{2}+\int \varepsilon p\left[(u-1)^{+}\right]^{p-1}|\nabla u|^{2} \phi^{2} \\
\leq 2 \varepsilon \int\left[(u-1)^{+}\right]^{p} \phi|\nabla \phi||\nabla u|+\int|f|\left[(u-1)^{+}\right]^{p} \phi^{2} \\
\leq \frac{p \varepsilon}{2} \int\left[(u-1)^{+}\right]^{p-1}|\nabla u|^{2} \phi^{2}+\frac{2 \varepsilon}{p} \int\left[(u-1)^{+}\right]^{p+1}|\nabla \phi|^{2} \\
+\frac{\kappa}{2 \varepsilon} \int\left[(u-1)^{+}\right]^{p+1} \phi^{2}+\frac{\varepsilon^{p} c(p)}{\kappa^{p}} \int|f|^{p+1} \phi^{2},
\end{gathered}
$$


which shows

$$
\frac{\kappa}{2 \varepsilon} \int\left[(u-1)^{+}\right]^{p+1} \phi^{2} \leq \frac{2 \varepsilon}{p} \int\left[(u-1)^{+}\right]^{p+1}|\nabla \phi|^{2}+\frac{\varepsilon^{p} c(p)}{\kappa^{p}} \int|f|^{p+1} \phi^{2} .
$$

Since $\|f\|_{L^{p+1}} \leq c(n, p)\|f\|_{W^{1, n}} \leq c(n, p) \lambda_{0}$ by the Sobolev inequality for any $p<\infty$, and since $|u|$ is bounded by $c_{0}$, we obtain

$$
\int_{B_{1-s}}\left[(u-1)^{+}\right]^{p+1} \leq c_{12}\left(s, p, c_{0}, \lambda_{0}, \kappa\right) \varepsilon^{p+1}
$$

by iterating the above estimate. To derive a contradiction, assume that $u\left(x_{0}\right)-1 \geq \varepsilon^{\eta}$ for some $x_{0} \in B_{1-s}$. By the gradient estimate $|\nabla u| \leq c_{5} \varepsilon^{-1}$ (as in the proof of (3.5)), thus for $y \in B_{\varepsilon^{1+\eta} / 2 c_{5}}\left(x_{0}\right)$,

$$
u(y)-1 \geq u\left(x_{0}\right)-1-\sup |\nabla u| \cdot \varepsilon^{1+\eta} / 2 c_{5} \geq \varepsilon^{\eta} / 2
$$

Then

$$
c_{12} \varepsilon^{p+1} \geq \int_{B_{\varepsilon^{1+\eta} / 2 c_{5}}\left(x_{0}\right)}\left[(u-1)^{+}\right]^{p+1} \geq \frac{\omega_{n} \varepsilon^{\eta(p+1)}}{2^{p+1}}\left(\varepsilon^{1+\eta} / 2 c_{5}\right)^{n} .
$$

The right-hand side is of order $\varepsilon^{p \eta+\eta(n+1)+n}$. Since $\eta<1$, for sufficiently large $p\left(p>\frac{\eta(n+1)+n-1}{1-\eta}\right)$ and small $\varepsilon$, the inequality cannot hold. This would be a contradiction. Hence we obtain (3.11). $u \geq-1-\varepsilon^{\eta}$ is proved similarly.

It is convenient to work in the $\varepsilon$-scale, namely, we consider $B_{3 d} \subset \widetilde{U}$, and consider the rescaled problem

$$
-\Delta u+W^{\prime}(u)=\varepsilon f \quad \text { on } B_{3 d / \varepsilon} .
$$

Without loss of generality, we choose a suitable unit so that $d=1$. Also we denote the rescaled discrepancy function by

$$
\xi=\frac{|\nabla u|^{2}}{2}-W(u)
$$

With this scale, we need to prove

$$
\sup _{B_{\varepsilon-1}} \xi \leq c_{1} \varepsilon^{3 / 5}
$$

In the following we use

$$
\xi_{G}(x)=\frac{|\nabla u|^{2}}{2}-W(u)-G(u)
$$

where $G: \mathbb{R} \rightarrow \mathbb{R}$ will be fixed shortly. We first obtain a differential inequality for $\xi_{G}$, cf. $[14,29,24]$. For the reader's convenience, we supply the proof. 
Lemma 3.8. On $|\nabla u|>0$,

$$
\begin{gathered}
\Delta \xi_{G}-\frac{2\left(W^{\prime}+G^{\prime}\right) \nabla u}{|\nabla u|^{2}} \cdot \nabla \xi_{G}+2 G^{\prime \prime} \xi_{G} \geq\left(G^{\prime}\right)^{2} \\
+G^{\prime} W^{\prime}-2 G^{\prime \prime}(W+G)+\varepsilon f\left(W^{\prime}+G^{\prime}\right)-\varepsilon \nabla f \cdot \nabla u .
\end{gathered}
$$

Proof. Compute

$$
\begin{aligned}
\Delta \xi_{G} & =\left|\nabla^{2} u\right|^{2}+\sum u_{x_{i}}(\Delta u)_{x_{i}}-W^{\prime \prime}|\nabla u|^{2}-W^{\prime} \Delta u-G^{\prime \prime}|\nabla u|^{2}-G^{\prime} \Delta u \\
& =\left|\nabla^{2} u\right|^{2}-\left(W^{\prime}+G^{\prime}\right) \Delta u-G^{\prime \prime}|\nabla u|^{2}-\varepsilon \nabla u \cdot \nabla f \\
& \left(\text { by } \Delta u=W^{\prime}-\varepsilon f\right)
\end{aligned}
$$$$
=\left|\nabla^{2} u\right|^{2}-\left(W^{\prime}+G^{\prime}\right)\left(W^{\prime}-\varepsilon f\right)-2 G^{\prime \prime}\left(G+W+\xi_{G}\right)-\varepsilon \nabla u \cdot \nabla f,
$$

where the last line is by substituting $|\nabla u|^{2}=2\left(G+W+\xi_{G}\right)$. On the other hand,

$$
\begin{aligned}
|\nabla u|^{2}\left|\nabla^{2} u\right|^{2} & \geq \sum_{j}\left(\sum_{i} u_{x_{i}} u_{x_{i} x_{j}}\right)^{2} \\
& =\sum_{j}\left(\left(\xi_{G}\right)_{x_{j}}+\left(W^{\prime}+G^{\prime}\right) u_{x_{j}}\right)^{2} \\
& \geq 2\left(W^{\prime}+G^{\prime}\right) \nabla u \cdot \nabla \xi_{G}+\left(W^{\prime}+G^{\prime}\right)^{2}|\nabla u|^{2} .
\end{aligned}
$$

We may then conclude (3.13).

Lemma 3.9. Suppose $0<\eta, \beta<1,0 \leq \iota \leq 1$ satisfy $2 \beta+\iota \geq 2 \eta$, $0<s<1,0<c_{11}<1$. Then, there exist $0<\varepsilon_{4}<\varepsilon_{3}, 0<c_{12}, c_{13}<\infty$ depending only on $\eta, \beta, \iota, s, c_{0} \lambda_{0}, c_{11}$, dist $(\tilde{U}, \partial U), n$ and $W$ with the following properties:

Suppose

(A) $u \in C^{3}\left(B_{\varepsilon^{-\beta}}\right), f \in C^{1}\left(B_{\varepsilon^{-\beta}}\right)$ and $\varepsilon \leq \varepsilon_{4}$ satisfy

$$
-\Delta u+W^{\prime}(u)=\varepsilon f
$$

on $B_{\varepsilon^{-\beta}}$ and

(B) $\sup _{B_{\varepsilon^{-\beta}}}|u| \leq 1+\varepsilon^{\eta} \sup _{B_{\varepsilon_{-\beta}}} \xi \leq c_{11} \varepsilon^{\iota}$.

Then,

$\sup _{B_{(1-s) \varepsilon^{-\beta}}} \xi \leq c_{12}\left\{\varepsilon^{1-\beta}\left(\|\nabla f\|_{L^{n}\left(B_{\varepsilon^{-\beta}}\right)}+\|f\|_{L^{n}\left(B_{\varepsilon^{-\beta}} \cap\left\{|f| \geq c_{13} \varepsilon^{\eta-1}\right\}\right)}\right)+\varepsilon^{\eta}\right\}$.

Proof. We choose $\tilde{\phi} \in C^{\infty}\left(B_{1}\right)$ such that

$$
\tilde{\phi}(x)= \begin{cases}1 & \text { on } B_{1} \backslash B_{1-\frac{s}{2}} \\ 0 & \text { on } B_{1-s}\end{cases}
$$


$0 \leq \tilde{\phi} \leq 1,|\nabla \tilde{\phi}|,\left|\nabla^{2} \tilde{\phi}\right|,|\nabla \tilde{\phi}| / \sqrt{\tilde{\phi}} \leq c_{s}$, where we denote generic positive constants depending only on $s$ and $n$ as $c_{s}$. For simplicity, we use the same notation $c_{s}$ for such constants. Then define

$$
\phi(x)=c_{11} \varepsilon^{\iota} \tilde{\phi}\left(\varepsilon^{\beta} x\right)
$$

for $x \in B_{\varepsilon^{-\beta}}$. With this definition and by the properties of $\tilde{\phi}$, we have

$$
|\nabla \phi| \leq c_{11} c_{s} \varepsilon^{\beta+\iota}, \quad\left|\nabla^{2} \phi\right| \leq c_{11} c_{s} \varepsilon^{2 \beta+\iota}, \quad|\nabla \phi| / \sqrt{\phi} \leq c_{11}^{1 / 2} c_{s} \varepsilon^{\beta+\frac{\iota}{2}} .
$$

Let $G(u)=c_{14} \varepsilon^{\eta}\left(1-(u-\gamma)^{2} / 8\right)$, where $c_{14} \geq 1$ will be determined later, and define

$$
\tilde{\xi}=\xi-G-\phi \quad\left(=\xi_{G}-\phi\right) .
$$

By restricting $\varepsilon$ depending only on $\gamma$ and by Assumption (B), we have $G>0$. We later use $G^{\prime} W^{\prime} \geq 0$ on $u \in[-1,1]$ as well as $G^{\prime \prime}<0$ for any $u$. On $B_{\varepsilon^{-\beta}} \backslash B_{\left(1-\frac{s}{2}\right) \varepsilon^{-\beta}}, \phi=c_{11} \varepsilon^{\iota}$, hence by (B), $\tilde{\xi} \leq 0$ there. Also, $\tilde{\xi}=\xi-G$ on $B_{(1-s) \varepsilon^{-\beta}}$ since $\phi=0$ there. In particular, if $\tilde{\xi} \leq 0$ on $B_{\varepsilon^{-\beta}}$, then $\xi \leq G \leq c_{14} \varepsilon^{\eta}$ on $B_{(1-s) \varepsilon^{-\beta}}$. In the following, we denote

$$
M=\sup _{B_{\varepsilon^{-\beta}}} \tilde{\xi}^{+}
$$

and assume that $M>0$ (or else, we are done). We next apply the Aleksandorov-Bakelman-Pucci maximum principle to $\tilde{\xi}^{+}$on $B_{\varepsilon^{-\beta}}$. Note that $\tilde{\xi}^{+}=0$ on $\partial B_{\varepsilon^{-\beta}}$. Let $\Gamma^{+}$be the upper contact set of the graph of $\tilde{\xi}^{+}$. Then, with the diameter of the domain being $2 \varepsilon^{-\beta}$, we have (see $[21$, Lemma 9.3])

$$
\frac{M}{2 \varepsilon^{-\beta}} \leq \frac{1}{n \omega_{n}^{1 / n}}\left(\int_{\Gamma^{+}}(-\Delta \tilde{\xi})^{n}\right)^{1 / n}
$$

Note that $\tilde{\xi}^{+}=\tilde{\xi}$ and $\Delta \tilde{\xi} \leq 0$ on $\Gamma^{+}$, and the slope of the support hyperplane is equal to the gradient of $\tilde{\xi}$. Since such hyperplanes have slope less than $M \varepsilon^{\beta} / s$, we have

$$
|\nabla \tilde{\xi}| \leq M \varepsilon^{\beta} / s \quad \text { on } \Gamma^{+} .
$$

Also, since $\tilde{\xi}^{+}=0$ on $B_{\varepsilon^{-\beta}} \backslash B_{\left(1-\frac{s}{2}\right) \varepsilon^{-\beta}}$, the height of the contact points of the graph of $\tilde{\xi}^{+}$and the supporting hyperplanes have a lower bound:

$$
\tilde{\xi}=\tilde{\xi}^{+} \geq \frac{s M}{2} \quad \text { on } \Gamma^{+} .
$$

In the following, we carefully estimate $-\Delta \tilde{\xi}$ from above. In doing so, we need to consider three cases, and define three subsets of $\Gamma^{+} \cap B_{\varepsilon^{-\beta}}$ 
PHASE FIELD MODEL

by

$$
\begin{gathered}
\mathcal{A}=\{x|| u(x) \mid \geq 1\}, \\
\mathcal{B}=\{x|(\gamma+1) / 2 \leq| u(x) \mid \leq 1\} \\
\mathcal{C}=\{x|| u(x) \mid \leq(\gamma+1) / 2\} .
\end{gathered}
$$

By (3.13), we have

$$
\begin{aligned}
& -\Delta \tilde{\xi}=-\Delta \xi_{G}+\Delta \phi \\
& \leq\left\{-\frac{2\left(W^{\prime}+G^{\prime}\right) \nabla u}{|\nabla u|^{2}} \cdot \nabla \xi_{G}\right\}+\left\{2 G^{\prime \prime} \xi_{G}-\left(G^{\prime}\right)^{2}\right\}+\left\{-G^{\prime} W^{\prime}+2 G^{\prime \prime}(W+G)\right\} \\
& +\left\{-\varepsilon f\left(W^{\prime}+G^{\prime}\right)+\Delta \phi\right\}+\{\varepsilon \nabla u \cdot \nabla f\} \equiv\{I\}+\{I I\}+\{I I I\}+\{I V\}+\{V\} \\
& (I) \text { Since } \xi_{G}=\tilde{\xi}+\phi \text { and using }|\nabla u|=\sqrt{2} \sqrt{\tilde{\xi}+W+G+\phi} \geq(\sqrt{\tilde{\xi}}+\sqrt{\phi}) \\
& I \leq \frac{2}{|\nabla u|}\left(\left|W^{\prime}\right|+c_{14} \varepsilon^{\eta}\right)|\nabla \tilde{\xi}+\nabla \phi| \\
& \leq 2\left(\left|W^{\prime}\right|+c_{14} \varepsilon^{\eta}\right)\left(\frac{|\nabla \tilde{\xi}|}{\sqrt{\tilde{\xi}}}+\frac{|\nabla \phi|}{\sqrt{\phi}}\right) \\
& \quad \leq 2\left(\left|W^{\prime}\right|+c_{14} \varepsilon^{\eta}\right)\left(\sqrt{\frac{2 M}{s^{3}}} \varepsilon^{\beta}+c_{11}^{1 / 2} c_{s} \varepsilon^{\beta+\frac{\iota}{2}}\right) \leq\left(\left|W^{\prime}\right|+c_{14} \varepsilon^{\eta}\right) c_{s} c_{11}^{1 / 2} \varepsilon^{\beta+\frac{\iota}{2}}
\end{aligned}
$$

The last two lines are by (3.15-17) as well as $M \leq c_{11} \varepsilon^{\iota}$. By separating into three cases, we find

$$
I \leq c_{s} c_{11}^{1 / 2} \varepsilon^{\beta+\frac{\iota}{2}} \times \begin{cases}\left(\sup \left|W^{\prime \prime}\right| \varepsilon^{\eta}+c_{14} \varepsilon^{\eta}\right) & \text { on } \mathcal{A} \\ \left(\left|W^{\prime}\right|+c_{14} \varepsilon^{\eta}\right) & \text { on } \mathcal{B} \\ \left(\sup \left|W^{\prime}\right|+c_{14} \varepsilon^{\eta}\right) & \text { on } \mathcal{C}\end{cases}
$$

$(I I)$ Since $\xi_{G}=\tilde{\xi}+\phi \geq 0$ on $\Gamma^{+}$and $G^{\prime \prime}<0$, we have

$$
I I \leq 0 \text {. }
$$

(III) By the choice of $G$, both terms are non-positive on $\mathcal{B}$ and $\mathcal{C}$. Moreover, since $G^{\prime \prime}=-\frac{c_{14}}{4} \varepsilon^{\eta}$ and $G \geq c_{14} \varepsilon^{\eta} / 2$ for $|u| \leq 1+\varepsilon^{\eta}$ for suitably small $\varepsilon$,

$$
I I I \leq-\frac{\left(c_{14} \varepsilon^{\eta}\right)^{2}}{8} \begin{cases}+c_{14} \varepsilon^{2 \eta} \sup \left|W^{\prime \prime}\right| & \text { on } \mathcal{A} \\ -c_{14} \varepsilon^{\eta} \frac{(1-\gamma)}{8}\left|W^{\prime}\right| & \text { on } \mathcal{B} \\ -\frac{c_{14}}{4} \varepsilon^{\eta} \min _{|u| \leq \frac{\gamma+1}{2}} W(u) & \text { on } \mathcal{C} .\end{cases}
$$

The first term comes from $G^{\prime \prime} G$. Note that $\min _{|u| \leq \frac{\gamma+1}{2}} W(u)>0$ is a strictly positive constant. 
(IV) By (3.11) and (3.15),

$$
\begin{aligned}
I V & \leq|f| \varepsilon\left(\left|W^{\prime}\right|+c_{14} \varepsilon^{\eta}\right)+c_{11} c_{s} \varepsilon^{2 \beta+\iota} \\
& \leq c_{11} c_{s} \varepsilon^{2 \beta+\iota}+ \begin{cases}\left(\varepsilon^{1+\eta} \sup \left|W^{\prime \prime}\right|+\varepsilon^{1+\eta} c_{14}\right)|f| & \text { on } \mathcal{A} \\
\left(\varepsilon\left|W^{\prime}\right|+c_{14} \varepsilon^{1+\eta}\right)|f| & \text { on } \mathcal{B}, \\
\left(\varepsilon \sup \left|W^{\prime}\right|+c_{14} \varepsilon^{1+\eta}\right)|f| & \text { on } \mathcal{C} .\end{cases}
\end{aligned}
$$

Next, we sum the four terms on each set and evaluate them from above. Note that the terms in $(I I I)$ are "good terms", giving the necessary negative contributions.

$(I+\cdots+I V$ on $\mathcal{A})$

$$
\begin{aligned}
I+\cdots+I V & \leq c_{14}^{2} \varepsilon^{2 \eta}\left(\frac{c_{s} c_{11}^{1 / 2}}{c_{14}^{2}} \sup \left|W^{\prime \prime}\right| \varepsilon^{\beta+\frac{\iota}{2}-\eta}+\frac{c_{s} c_{11}^{1 / 2}}{c_{14}} \varepsilon^{\beta+\frac{\iota}{2}-\eta}-\frac{1}{8}\right. \\
& \left.+\frac{\sup \left|W^{\prime \prime}\right|}{c_{14}}+\frac{c_{11} c_{s}}{c_{14}^{2}} \varepsilon^{2 \beta+\iota-2 \eta}\right)+\left(\varepsilon^{1+\eta} \sup \left|W^{\prime \prime}\right|+\varepsilon^{1+\eta} c_{14}\right)|f| .
\end{aligned}
$$

Since $2 \eta \leq 2 \beta+\iota$, we may restrict $c_{14}$ large depending only on $c_{11}, c_{s}, W$ so that

$$
\begin{aligned}
I+\cdots+I V & \leq-\frac{c_{14}^{2}}{16} \varepsilon^{2 \eta}+\left(\varepsilon^{1+\eta} \sup \left|W^{\prime \prime}\right|+\varepsilon^{1+\eta} c_{14}\right)|f| \\
& \leq c_{15} \varepsilon^{1+\eta}|f| \cdot \chi_{\left\{|f| \geq c_{16} \varepsilon^{\eta-1}\right\}}
\end{aligned}
$$

for suitable choices of $c_{15}, c_{16}=c\left(c_{11}, c_{s}, W\right)>0$. Here, $\chi_{A}$ denotes the characterictic function of $A$.

$(I+\cdots+I V$ on $\mathcal{B})$

$$
\begin{aligned}
I+\cdots+I V & \leq\left|W^{\prime}\right| c_{14} \varepsilon^{\eta}\left(\frac{c_{s} c_{11}^{1 / 2}}{c_{14}} \varepsilon^{\beta+\frac{\iota}{2}-\eta}-\frac{1-\gamma}{8}\right) \\
& +\left(c_{14} \varepsilon^{\eta}\right)^{2}\left(\frac{c_{s} c_{11}^{1 / 2}}{c_{14}} \varepsilon^{\beta+\frac{\iota}{2}-\eta}-\frac{1}{8}+\frac{c_{11} c_{s}}{c_{14}^{2}} \varepsilon^{2 \beta+\iota-2 \eta}\right) \\
& +\left(\varepsilon\left|W^{\prime}\right|+c_{4} \varepsilon^{1+\eta}\right)|f|
\end{aligned}
$$

Again with $2 \eta \leq 2 \beta+\iota$ and restricting $c_{14}$ large, we have

$$
\begin{aligned}
I+\cdots+I V & \leq-\frac{1-\gamma}{16} c_{14} \varepsilon^{\eta}\left|W^{\prime}\right|-\frac{c_{14}^{2}}{16} \varepsilon^{2 \eta}+\left(\varepsilon\left|W^{\prime}\right|+c_{14} \varepsilon^{1+\eta}\right)|f| \\
& \leq c_{15} \varepsilon|f| \cdot \chi_{\left\{|f| \geq c_{16} \varepsilon^{\eta-1}\right\}}
\end{aligned}
$$


PHASE FIELD MODEL

where $c_{15}, c_{16}$ are chosen appropriately again.

$(I+\cdots+I V$ on $\mathcal{C})$

$$
\begin{aligned}
I+\cdots+I V & \leq c_{14} \varepsilon^{\eta}\left(\frac{c_{s} c_{11}^{1 / 2} \sup \left|W^{\prime}\right|}{c_{14}} \varepsilon^{\beta+\frac{\iota}{2}-\eta}+c_{s} c_{11}^{1 / 2} \varepsilon^{\beta+\frac{\iota}{2}}\right. \\
& \left.-\frac{1}{4} \min _{|u| \leq \frac{\gamma+1}{2}} W(u)+\frac{c_{11} c_{s}}{c_{14}} \varepsilon^{2 \beta+\iota-\eta}\right)+\left(\varepsilon \sup \left|W^{\prime}\right|+c_{14} \varepsilon^{1+\eta}\right)|f| .
\end{aligned}
$$

Using $0<\eta \leq \beta+\frac{\iota}{2}$ and restricting $\varepsilon$ small and $c_{14}$ large depending only on $\eta, W, c_{11}$ and $c_{s}$, we have

$$
\begin{aligned}
I+\cdots+I V & \leq-\frac{c_{14}}{8} \varepsilon^{\eta} \min _{|u| \leq \frac{\gamma+1}{2}} W(u)+\left(\varepsilon \sup \left|W^{\prime}\right|+c_{14} \varepsilon^{1+\eta}\right)|f| \\
& \leq c_{15} \varepsilon|f| \cdot \chi_{\left\{|f| \geq c_{16} \varepsilon^{\eta-1}\right\}}
\end{aligned}
$$

for suitable choices of $c_{15}, c_{16}$.

$(I+\cdots+V)$ Combining $(3.18-20)$ and $|\nabla u| \leq c_{5}$, we have

$$
I+\cdots+V \cdot \leq c_{5} \varepsilon|\nabla f|+c_{15} \varepsilon|f| \cdot \chi_{\left\{|f| \geq c_{16} \varepsilon^{\eta-1}\right\}}
$$

on $\Gamma^{+}$. Thus we have

$$
\left(\int_{\Gamma^{+}}(-\Delta \tilde{\xi})^{n}\right)^{1 / n} \leq c_{5} \varepsilon\|\nabla f\|_{L^{n}\left(\Gamma^{+}\right)}+c_{15} \varepsilon\|f\|_{L^{n}\left(\Gamma^{+} \cap\left\{|f| \geq c_{16} \varepsilon^{\eta-1}\right\}\right)} .
$$

Since

$$
\sup _{B_{\varepsilon^{-\beta}(1-s)}} \tilde{\xi} \leq M \leq \frac{2 \varepsilon^{-\beta}}{n \omega_{n}^{1 / n}}\left(\int_{\Gamma^{+}}(-\Delta \tilde{\xi})^{n}\right)^{1 / n},
$$

$\tilde{\xi}=\xi-G$ and $G \leq c_{14} \varepsilon^{\eta}$, we have the desired estimate by suitably choosing $c_{12}$ and $c_{13}$.

Here we give a proof for (3.12). By the Sobolev inequality (applied to the original scale), for any $p<\infty$, we have

$$
\left(\varepsilon^{n} \int_{B_{(1-s) \varepsilon^{-1}}}|f|^{p}\right)^{1 / p} \leq c(p, n, s) \lambda_{0}
$$

In particular, for any $t \geq 1$,

$$
\begin{gathered}
\left(\int_{B_{(1-s) \varepsilon-1} \cap\{|f| \geq t\}}|f|^{n}\right)^{1 / n} \leq t^{1-\frac{p}{n}}\left(\int_{B_{(1-s) \varepsilon}-1}|f|^{p}\right)^{1 / n} \\
\leq t^{1-\frac{p}{n}} \varepsilon^{-\frac{1}{p}} c(p, n, s) \lambda_{0} .
\end{gathered}
$$




\section{YOSHIHIRO TONEGAWA}

Thus, in if $t=\varepsilon^{\eta-1}$, with $0<\eta$, with an appropriately large $p$, the (LHS) of (3.14) is bounded in terms of $\lambda_{0}$ uniformly for all small $\varepsilon$. Now we use (3.14) with $\eta=\beta=1 / 2$ and $\iota=0$ to any ball $B_{\varepsilon^{-1 / 2}}(x) \subset B_{\varepsilon^{-1}}$. The condition (B) is satisfied for a suitable constant $c_{11}$, and $2 \beta+\iota \geq 2 \eta$ is satisfied. Thus we have

$$
\sup _{B_{(1-s) \varepsilon^{-1}}} \xi \leq c_{12}\left(\varepsilon^{1 / 2}+\varepsilon^{1 / 2}\|\nabla f\|_{L^{n}}+\varepsilon^{1 / 2}\|f\|_{L^{n}\left(B_{\varepsilon^{-1}} \cap\left\{|f| \geq c_{13} \varepsilon^{-1 / 2}\right\}\right)}\right) .
$$

Next we apply Lemma 3.9 again with $\eta=3 / 5, \beta=2 / 5, \iota=1 / 2$ and the new $c_{11}$. With this choice, $2 \eta \leq 2 \beta+\iota$ is satisfied. Since $1-\beta=\eta=3 / 5$, we obtain (3.12).

Remark. The exponent $3 / 5$ in (3.12) is simply a convenient choice for us, but the argument works just as well, as long as the exponent is strictly larger than $1 / 2$. If we assume that $f \in W^{1, p}$ for $p$ sufficiently larger than $n$ (for example $p \geq 2 n$ ), then we can prove a better estimate with the exponent equal 1. As far as we can see, however, we could not obtain such estimate for the case $p=n$.

\section{RECTIFIABILITY AND INTEGRALITY OF THE LIMIT VARIFOLD}

Proposition 4.1. There exist constants $0<D_{1} \leq D_{2}<\infty$ and $r_{0}>0$ which depend only on $\lambda_{0}, c_{0}, E_{0}$, $\operatorname{dist}(\tilde{U}, \partial U)$ and $W$, such that

$$
D_{1} r^{n-1} \leq \mu\left(B_{r}(x)\right) \leq D_{2} r^{n-1}
$$

for all $0<r<r_{0}, x \in \operatorname{supp} \mu \cap \tilde{U}$ and $B_{r}(x) \subset \tilde{U}$.

Proof. The existence of $D_{2}$ is immediate from (3.10).

To establish the lower bound, let $x \in \operatorname{supp} \mu \cap \tilde{U}$.

Claim. On passing to a subsequence there exist $x_{i} \in \tilde{U}$ such that $u^{i}\left(x_{i}\right) \in[-\alpha, \alpha]$ and $x_{i} \rightarrow x$ as $i \rightarrow \infty$.

Proof of Claim. Suppose the converse. Then there exists some $s>0$ such that $B_{s}(x) \subset \tilde{U}$ and $B_{s}(x) \cap\left\{\left|u^{i}\right| \leq \alpha\right\}=\emptyset$ for all sufficiently large $i$. For each such $i$ either $u^{i}>\alpha$ on $B_{s}(x)$ or $u^{i}<-\alpha$ on $B_{s}(x)$. If $u^{i}>\alpha$, by using the argument in Proposition 3.7 one shows that $u^{i} \in\left[1-\varepsilon_{i}^{3 / 4}, 1+\varepsilon_{i}^{3 / 4}\right]$ on $B_{s / 2}(x)$ for all sufficiently large $i$. Similarly, if $u^{i}<-\alpha$, then $u^{i} \in\left[-1-\varepsilon_{i}^{3 / 4},-1+\varepsilon_{i}^{3 / 4}\right]$ on $B_{s / 2}(x)$. This implies $W\left(u^{i}\right)=O\left(\varepsilon_{i}^{3 / 2}\right)$ and thus $\frac{W\left(u^{i}\right)}{\varepsilon_{i}} \rightarrow 0$ uniformly on $B_{s / 2}(x)$ as $i \rightarrow \infty$.

For $\frac{\varepsilon_{i}\left|\nabla u^{i}\right|^{2}}{2}$, by using the argument in Proposition 3.5 (estimate on $\mathcal{C})$, one shows that

$$
\int_{B_{s / 2}(x)} \varepsilon_{i}\left|\nabla u^{i}\right|^{2} \leq O\left(\varepsilon_{i}^{2}\right) \rightarrow 0
$$


as $i \rightarrow \infty$. Hence we may conclude that $\mu\left(B_{s / 2}(x)\right)=0$, which is a contradiction to $x \in \operatorname{supp} \mu$. This ends the proof of the claim.

For any $x \in \tilde{U} \cap \operatorname{supp} \mu, B_{r}(x) \subset \tilde{U}$ and $r \leq r_{0}$, Proposition 3.4 and 3.6 show that

$$
\begin{aligned}
\frac{1}{r^{n-1}} \mu\left(B_{r}(x)\right) & \geq \lim _{i \rightarrow \infty} \frac{1}{r^{n-1}} \int_{B_{r / 2}\left(x_{i}\right)} \frac{\varepsilon_{i}\left|\nabla u^{i}\right|^{2}}{2}+\frac{W\left(u^{i}\right)}{\varepsilon_{i}} \\
& \geq \liminf 2^{1-n} e^{c_{7} r / 2}\left\{-c_{9} r^{1 / 2}+e^{-c_{7} c_{3} \varepsilon_{i}^{2 / 5}} E\left(c_{3} \varepsilon_{i}^{2 / 5}, x_{i}\right)\right\} \\
& \geq 2^{1-n} e^{-c_{7} r / 2}\left\{-c_{9} r^{1 / 2}+e^{-c_{7} c_{3}} c_{4}\right\} .
\end{aligned}
$$

Thus, by suitably restricting $r$, we prove the existence of $D_{1}$.

Proposition 4.2. Either $u^{i} \rightarrow+1$ or $u^{i} \rightarrow-1$ uniformly on each compact subset of $U \backslash$ supp $\|V\|$. In particular, supp $\left\|\partial\left\{u^{\infty}=1\right\}\right\| \subset$ supp $\|V\|$. The terms $\frac{\varepsilon_{i}}{2}\left|\nabla u^{i}\right|^{2}$ and $\varepsilon_{i}^{-1} W\left(u^{i}\right)$ converge uniformly to zero on compact subsets of $U \backslash \operatorname{supp}\|V\|$.

Proof. This follows immediately from the argument for the previous proposition.

Let

$$
\xi^{i}=\frac{\varepsilon_{i}\left|\nabla u^{i}\right|^{2}}{2}-\frac{W\left(u^{i}\right)}{\varepsilon_{i}},
$$

and define (passing to a subsequence if necessary) the measure $|\xi|$ on $U$ by

$$
|\xi|(\phi)=\lim _{i \rightarrow \infty} \int\left|\xi^{i}\right| \phi
$$

for nonnegative $\phi \in C_{c}(U)$. Thus $|\xi|$ is the measure theoretic limit of the absolute values of the discrepancy functions.

Proposition 4.3. $|\xi|$ is the zero measure and so $\xi^{i} \rightarrow 0$ in $L_{l o c}^{1}(U)$. Moreover, both $\frac{\varepsilon_{i}}{2}\left|\nabla u^{i}\right|^{2}-\left|\nabla w^{i}\right|$ and $\frac{1}{\varepsilon_{i}} W\left(u^{i}\right)-\left|\nabla w^{i}\right|$ also converge to zero in $L_{\text {loc }}^{1}(U)$.

Proof. First we claim that

$$
\liminf _{r \rightarrow 0} \frac{1}{r^{n-1}}|\xi|\left(B_{r}(x)\right)=0
$$

for all $x \in \operatorname{supp}|\xi| \cap \tilde{U}$. Otherwise, there would exist $x \in \operatorname{supp}|\xi| \cap \widetilde{U}$, $R>0$ and $b>0$ such that $R \leq r_{0}$ and $|\xi|\left(B_{r}(x)\right) \geq b r^{n-1}$ for all $0<r \leq R$. Define

$$
r_{1}=\min \left\{b\left(8 D_{2} c_{7}+c_{8}\right)^{-1}, R\right\}
$$


YOSHIHIRO TONEGAWA

$$
r_{2}=r_{1} \min \left\{\exp \left[-4 b^{-1}\left(c_{9} r_{1}^{1 / 2}+4 D_{2} \exp \left[c_{7} r_{1}\right]\right)\right], 1 / 2\right\} .
$$

By Proposition 4.1 and the definition of $|\xi|$, we may choose a large enough $i$ such that $c_{3} \varepsilon_{i}^{2 / 5} \leq r_{2}$ and

$$
\frac{1}{\tau^{n-1}} \int_{B_{\tau}(x)} \frac{\varepsilon_{i}\left|\nabla u^{i}\right|^{2}}{2}+\frac{W\left(u^{i}\right)}{\varepsilon_{i}} \leq 2 D_{2}, \quad \frac{1}{\tau^{n-1}} \int_{B_{\tau}(x)}\left|\xi^{i}\right| \geq \frac{b}{2}
$$

for all $r_{2} \leq \tau \leq r_{1}$. By Proposition 3.5 and the definition of $r_{1}$,

$$
\begin{gathered}
\frac{1}{\tau^{n-1}} \int_{B_{\tau}(x)}\left(\frac{W\left(u^{i}\right)}{\varepsilon_{i}}-\frac{\varepsilon_{i}\left|\nabla u^{i}\right|^{2}}{2}\right)^{+} \\
\geq \frac{1}{\tau^{n-1}} \int_{B_{\tau}(x)}\left|\xi^{i}\right|-\left(c_{7} E\left(u^{i}, \tau, x\right)+c_{8}\right) \tau \geq \frac{b}{2}-\left(c_{7} 2 D_{2}+c_{8}\right) r_{1} \geq \frac{b}{4}
\end{gathered}
$$

for all $r_{2} \leq \tau \leq r_{1}$. By Proposition 3.6,

$$
\begin{gathered}
2 D_{2} \geq \frac{1}{r_{1}^{n-1}} \int_{B_{r_{1}}(x)} \frac{\varepsilon_{i}\left|\nabla u^{i}\right|^{2}}{2}+\frac{W\left(u^{i}\right)}{\varepsilon_{i}} \\
\geq e^{-c_{7} r_{1}}\left(\frac{b}{4} \int_{r_{2}}^{r_{1}} \frac{d \tau}{\tau}-c_{9} r_{1}^{1 / 2}\right)=e^{-c_{7} r_{1}}\left(\frac{b}{4} \ln \left(\frac{r_{1}}{r_{2}}\right)-c_{9} r_{1}^{1 / 2}\right) \geq 4 D_{2},
\end{gathered}
$$

which is a contradiction, so we have proved (4.1).

Combined with Proposition 4.1 and $\operatorname{supp}|\xi| \subset \operatorname{supp} \mu$, we have

$$
\liminf _{r \rightarrow 0} \frac{|\xi|\left(B_{r}(x)\right)}{\mu\left(B_{r}(x)\right)}=0
$$

for all $x \in \operatorname{supp}|\xi|$. A standard result in measure theory then shows that $|\xi|=0$.

It follows that $\xi^{i} \rightarrow 0$ in $L_{\text {loc }}^{1}(U)$.

By completing the square and using $2\left|\nabla w^{i}\right|=\sqrt{2 W\left(u^{i}\right)}\left|\nabla u^{i}\right|$, we see that

$$
\begin{aligned}
\left.\left|\frac{\varepsilon_{i}}{2}\right| \nabla u^{i}\right|^{2}+\frac{W\left(u^{i}\right)}{\varepsilon_{i}}-2\left|\nabla w^{i}\right| \mid & =\left(\sqrt{\frac{\varepsilon_{i}}{2}}\left|\nabla u^{i}\right|-\sqrt{\frac{W\left(u^{i}\right)}{\varepsilon_{i}}}\right)^{2} \\
& \leq\left|\frac{\varepsilon_{i}\left|\nabla u^{i}\right|^{2}}{2}-\frac{W\left(u^{i}\right)}{\varepsilon_{i}}\right|=\left|\xi^{i}\right| .
\end{aligned}
$$

This implies the remaining claims in the proposition.

Proposition 4.4. The limit varifold $V$ satisfies $\|V\|=\frac{1}{2} \mu$ and is rectifiable. The first variation of $V$ is given by

$$
\delta V(g)=\frac{1}{2} \int_{U} u^{\infty} \operatorname{div}\left(f^{\infty} g\right)=-\int_{M^{\infty}} f^{\infty} g \cdot \nu^{\infty} d \mathcal{H}^{n-1}
$$


for any $g \in C_{c}^{1}\left(U ; \mathbb{R}^{n}\right)$, where $M^{\infty} \subset$ supp $\|V\|$ is the reduced boundary of $\left\{u^{\infty}=1\right\}$ and $f^{\infty}$ on $M^{\infty}$ is the trace of $f^{\infty} \in W^{1, n}(U)$. The generalized mean curvature vector $H$ is given by

$$
H(x)= \begin{cases}\frac{f^{\infty}(x)}{\theta(x)} \nu^{\infty}(x) & \mathcal{H}^{n-1} \text { a.e. } x \in M^{\infty}, \\ 0 & \mathcal{H}^{n-1} \text { a.e. } x \in \text { supp }\|V\| \backslash M^{\infty},\end{cases}
$$

where $\theta$ is the density function for $\|V\|$. Moreover, $f^{\infty}\left\lfloor M^{\infty} \in L_{l o c}^{p}\left(U, \mathcal{H}^{n-1}\right)\right.$ for any $1 \leq p<\infty$.

Proof. Since $\|V\|=\lim \left\|V^{i}\right\|$ and $\left\|V^{i}\right\|=\left|\nabla w^{i}\right| d \mathcal{L}^{n}$, it follows from Proposition 4.3 and the definition of $\mu$ that $\frac{1}{2} \mu=\|V\|$. Next we rearrange terms in (3.2) and using $\frac{u_{x_{j}}^{i}}{\left|\nabla u^{i}\right|}=\frac{w_{x_{j}}^{i}}{\left|\nabla w^{i}\right|}$, we have

$$
\begin{gathered}
\int\left(\operatorname{div} g-\sum_{j, k} \frac{w_{x_{j}}^{i}}{\left|\nabla w^{i}\right|} \frac{w_{x_{k}}^{i}}{\left|\nabla w^{i}\right|} g_{x_{k}}^{j}\right) \varepsilon_{i}\left|\nabla u^{i}\right|^{2} \\
=\int\left\{\left(\frac{\varepsilon_{i}\left|\nabla u^{i}\right|^{2}}{2}-\frac{W\left(u^{i}\right)}{\varepsilon_{i}}+u^{i} f^{i}\right) \operatorname{div} g+u^{i} g \cdot \nabla f^{i}\right\}
\end{gathered}
$$

for any $g \in C_{c}^{1}(U)$. Since $\varepsilon_{i}\left|\nabla u^{i}\right|^{2}-2\left|\nabla w^{i}\right|$ and $\xi^{i}$ converge to $0, u^{i}$ converges to $u^{\infty}$ strongly in $L^{p}$ for any $1<p<\infty$ and $f^{i}$ converges weakly in $W^{1, n}$ to $f^{\infty}$, we have

$$
\delta V(g)=\frac{1}{2} \int u^{\infty}\left(f^{\infty} \operatorname{div} g+g \cdot \nabla f^{\infty}\right) .
$$

Here we used (2.2), and note that $V^{i}$ converges to $V$ in the sense of varifold, hence $\delta V^{i}(g) \rightarrow \delta V(g)$. To justify the integration by parts for $f^{\infty} g$, we use the following theorem due to Meyers and Ziemer ([28], see also [36, Theorem 1.3]).

Theorem([44, Theorem 5.12.4]) Let $\mu$ be a positive Radon measure on $\mathbb{R}^{n}$ satisfying

$$
K(\mu) \equiv \sup _{x \in \mathbb{R}^{n}, r>0} \frac{1}{r^{n-1}} \mu\left(B_{r}(x)\right)<\infty
$$

Then,

for all $\phi \in C_{c}^{1}\left(\mathbb{R}^{n}\right)$.

$$
\left|\int_{\mathbb{R}^{n}} \phi d \mu\right| \leq c(n) K(\mu) \int_{\mathbb{R}^{n}}|\nabla \phi| d \mathcal{L}^{n}
$$

Since $\mathcal{H}^{n-1}\left\lfloor M^{\infty} \leq \frac{\omega_{n-1}}{2 D_{1}}\|V\|\right.$ and $\|V\|\left(B_{r}(x)\right) \leq \frac{D_{2} r^{n-1}}{2}$, we have $K\left(\mathcal{H}^{n-1}\left\lfloor M^{\infty} \cap \widetilde{U}\right) \leq D_{2} D_{1}^{-1} \omega_{n-1} / 4\right.$. Thus

$$
\left|\int_{M^{\infty}} \phi d \mathcal{H}^{n-1}\right| \leq c(n) D_{2} D_{1}^{-1} \int_{U}|\nabla \phi| \text {. }
$$




\section{YOSHIHIRO TONEGAWA}

for all $\phi \in C_{c}^{1}(\widetilde{U})$. Using this and smoothly approximating $f^{\infty}$, we obtain

$$
-\int_{M^{\infty}} f^{\infty} g \cdot \nu d \mathcal{H}^{n-1}=\frac{1}{2} \int_{U} \operatorname{div}\left(f^{\infty} g\right) u^{\infty}
$$

for all $g \in C_{c}^{1}\left(\widetilde{U} ; \mathbb{R}^{n}\right)$. Moreover, for any $1 \leq p<\infty$,

$$
\begin{gathered}
\int_{M^{\infty} \cap \widetilde{U}}\left|f^{\infty}\right|^{p} d \mathcal{H}^{n-1} \leq c(n) D_{2} D_{1}^{-1} \int_{U} p\left|f^{\infty}\right|^{p-1}\left|\nabla f^{\infty}\right| \\
\leq c(n, p) D_{2} D_{1}^{-1}\left\|f^{\infty}\right\|_{W^{1, n}(U)}^{p}<\infty
\end{gathered}
$$

by the Sobolev inequality. Since $|\delta V(g)| \leq \sup |g| \int_{M^{\infty} n \widetilde{U}}\left|f^{\infty}\right| d \mathcal{H}^{n-1}$ for all $g \in C_{c}^{1}\left(\widetilde{U} ; \mathbb{R}^{n}\right),\|\delta V(g)\|$ defines a Radon measure on $U$. By the lower density estimate bound of $\|V\|$ and Allard's rectifiability theorem $[1,5.5$.(1)], we can conclude that $V$ is rectifiable. It also follows that $\|\delta V\|$ is absolutely continuous with respect to $\mathcal{H}^{n-1}\left\lfloor M^{\infty}\right.$, and hence with respect to $\|V\|$ as well. Thus,

$$
\delta V(g)=-\int g \cdot H d\|V\|=-\int_{\text {supp }\|V\|} g \cdot H \theta d \mathcal{H}^{n-1} .
$$

The expression for $H$ follows from the second expression for $\delta V(g)$ in the statement of the proposition.

Note that this proves that $H \in L_{\text {loc }}^{p}\left(U ; \mathbb{R}^{n}\right)$ with respect to $\|V\|$ as well for any $1 \leq p<\infty$. By the standard theory for varifold with its mean curvature in $L^{p}, p \geq n-1$, the density function $\theta$ is well defined everywhere on $\operatorname{supp}\|V\|$ and upper-semicontinuous on $U$. This fact also follows directly from Proposition 3.6.

Next, we prove that $\theta(x)=N \sigma$ for some positive integer $\mathcal{H}^{n-1}$ a.e. on $\operatorname{supp}\|V\|$. With modifications, the line of proof is very similar to that of [24, Section 5], so we point out the difference so that the reader may follow the proof. There are two points which must be dealt with: the first point is that we only have $f \in W^{1, n}$ so that $f$ is "almost bounded" but not quite, and the second is that the discrepancy function has an estimate which is not as good as the case in [24], where we had $\xi \leq c \varepsilon$. These points can be resolved by replacing the pointwise estimates in [24] by suitable integral estimates.

The first proposition shows that there is only small energy uniformly in $\varepsilon$ in the region $\{u \approx \pm 1\}$.

Proposition 4.5. ([24, Proposition 5.1.]) Assume B is true with u, $\varepsilon, f$ and $B_{3}$, and suppose $s>0$. Then there exist positive constants $b$ 
PHASE FIELD MODEL

and $\varepsilon_{5}$ depending only on $\lambda_{0}, c_{0}, E_{0}, W$ and $s$ such that

$$
\int_{B_{1} \cap\{|u| \geq 1-b\}} \frac{W(u)}{\varepsilon} \leq s
$$

whenever $\varepsilon \leq \varepsilon_{5}$.

Proof. We use two lemmas, [24, Lemma 5.2, 5.3]. For the first lemma, one only need to use the ABP estimate instead of the pointwise estimate. Since it is a straightforward modification, we omit the proof. The rest of the proof goes through with minor modifications of constants.

The second proposition deals with the "cutting" the varifold horizontally into stacked single-layered interfaces. Define $T: \mathbb{R}^{n} \rightarrow \mathbb{R}^{n-1}$ by $T(x)=\left(x_{1}, \cdots, x_{n-1}\right)$. Also define $\nu=\left(\nu_{1}, \cdots, \nu_{n}\right)=\frac{\nabla u}{|\nabla u|}$ whenever $|\nabla u| \neq 0$ and $\nu=0$ when $|\nabla u|=0$. We additionally define

$$
e_{\varepsilon}=\frac{\varepsilon|\nabla u|^{2}}{2}+\frac{W(u)}{\varepsilon}, \quad \xi_{\varepsilon}=\frac{\varepsilon|\nabla u|^{2}}{2}-\frac{W(u)}{\varepsilon} .
$$

In [24, Proposition 5.5], one needs to change hypothesis (2).

Proposition 4.6. Corresponding to each $R, E_{0}, s$ and $N$ such that $0<R<\infty, 0<E_{0}<\infty, 0<s<1$ and $N$ is a positive integer, there exists $\eta>0$ with the following property:

Assume the following:

(1) $Y \subset \mathbb{R}^{n}$ has no more than $N+1$ elements, $T(y)=0$ for all $y \in Y$, $a>0,|y-z|>3 a$ for all $y, z \in Y$ and diameter $Y \leq \eta R$.

(2) On $\left\{x \in \mathbb{R}^{n} \mid \operatorname{dist}(x, Y)<R\right\}$, u satisfies (1.2) with $\|f\|_{W^{1, n}} \leq \eta$, $|u| \leq 2$ and

$$
\int_{a}^{R} \frac{d r}{r^{n}} \int_{B_{r}(x)}\left(\frac{\varepsilon\left|\nabla u^{2}\right|}{2}-\frac{W(u)}{\varepsilon}\right)^{+} \leq \eta R \quad \text { for each } x \in Y .
$$

(3) For each $y \in Y$ and $a \leq r \leq R$,

$$
\begin{gathered}
\int_{B_{r}(y)}\left|\xi_{\varepsilon}\right|+\left(1-\left(\nu_{n}\right)^{2}\right) \varepsilon|\nabla u|^{2} d y \leq \eta r^{n-1} \\
\int_{B_{r}(y)} \varepsilon|\nabla u|^{2} \leq E_{0} r^{n-1}
\end{gathered}
$$

Then we have

$$
\sum_{y \in Y} \frac{1}{a^{n-1}} \int_{B_{a}(y)} e_{\varepsilon} \leq s+\frac{1+s}{R^{n-1}} \int_{\{x \mid \operatorname{dist}(Y, x)<R\}} e_{\varepsilon} .
$$




\section{YOSHIHIRO TONEGAWA}

For the proof, one also modify the hypothesis (4) in [24, Lemma 5.4] just like above hypothesis (2). With this, the proof of Lemma 5.4 goes through with minor changes, and hence above proposition follows.

The third proposition deals with the $\varepsilon$-scale. Here, one does need a pointwise estimate on $\xi_{\varepsilon}$, but $\xi_{\varepsilon} \leq c_{1} \varepsilon^{-2 / 5}$ is sufficient.

Proposition 4.7. Given $0<s<1$ and $0<b<1$, there exist $0<\eta<$ 1 and $1<L<\infty$ depending also on $W$ with the following property: Assume $0<\varepsilon<1$ and $u$ satisfies (1.2) and $\xi_{\varepsilon} \leq \eta \varepsilon^{-1}$ on $B_{4 \varepsilon L}(0)$ with $\|f\|_{W^{1, n}} \leq \eta,|u(0)| \leq 1-b$, and

$$
\int_{B_{4 \varepsilon L(0)}}\left(\left|\xi_{\varepsilon}\right|+\left(1-\left(\nu_{n}\right)^{2}\right) \varepsilon|\nabla u|^{2}\right) \leq \eta(4 \varepsilon L)^{n-1}
$$

Then, we have $T^{-1}(0) \cap\left\{x \in B_{3 L \varepsilon}(0) \mid u(x)=u(0)\right\}=\{0\}$ and

$$
\left|\frac{1}{\omega_{n-1}(L \varepsilon)^{n-1}} \int_{B_{L \varepsilon}(0)} e_{\varepsilon}-2 \sigma\right| \leq s
$$

We only point out that the place $\xi_{\varepsilon} \leq \eta$ was used in the proof of $[24$, Propostion 5.6] is where we wanted to conclude $|u| \leq 1-\tilde{b}$ on $B_{4 L}(0)$ by suitably restricting $\eta$. But this can be done by having only $\xi_{\varepsilon} \leq \eta \varepsilon^{-1}$ in fact. Also, instead of $C^{2}$ estimate for $u$ (or $z$ ), we only need $C^{1, \beta}$ estimate for some $\beta>0$, which is available even if $f \in W^{1, n}$.

To end the proof of Theorem 1, we point out that the main difference from that of [24] for the proof is the condition (2) of Proposition 4.6. For this, note that the constants $c_{1}, c_{7}$ and $c_{8}$ in Proposition 3.2 and 3.5 of this paper scale like $r$, hence they are small constants in the blowup argument. It is not hard, then, to verify above Prop. 4.6. (2) using Prop. 3.2 and 3.5 for a rescaled sequence of solutions. The argument in [24] then shows that the density of the limit varifold $\|V\|$ is $N \sigma$, where $N$ is an integer, $\mathcal{H}^{n-1}$ a.e. on the support of $\|V\|$.

\section{CONCLUDING REMARKS AND APPLICATIONS}

5.1. On the Sobolev norm of chemical potential. In this paper we consider the situation where we control the $W^{1, n}$ norm of chemical potential $f$ as $\varepsilon \rightarrow 0$. We encounter a serious difficulty to relax the control when we estimate the supremum bound on $\frac{\varepsilon}{2}|\nabla u|^{2}-\frac{W}{\varepsilon}$. As noted in the beginning, it is conjectured that a control of $W^{1, p}$ for some $p>n / 2$ should be sufficient to obtain our result, with the limit mean curvature belonging to $L^{q}$ space for a smaller $q$. This follows from the following huristic argument: if the interface $M$ is $C^{1}$ hypersurface and if $H$ belongs to $W^{1, p}(U)$ for some $p>n / 2$, the trace of $H$ on $M$ belongs to $L^{q}(M)$ for $q>n-1$. If the mean curvature of $M$ is given by $H$, the 
well-known result on the regularity of integral varifold [1] shows that the monotonicity formula for the scaled energy ( $n-1$-dimensional area) holds for $M$. Since $f$ roughly corresponds to the mean curvature field of the interface, one expects that $f$ being in $W^{1, p}(U)$ for some $p>n / 2$ may be sufficient to obtain a monotonicity type formula, which may also prove all the subsequent rectifiability and integrality of the limit varifold. Quite relevant to this point are recent articles by Schätzle $[36,37]$, where he studied convergence of integral varifolds with their mean curvature given by Sobolev functions in $W^{1, p}(U), p>n / 2$. His results also sugget strongly that the multiplicity of the limit varifold in this paper is $\mathcal{H}^{n-1}$ a.e. $\sigma$, namely, no folding, on $M^{\infty} \cap\left\{f^{\infty} \neq 0\right\}$. Geometrically, if there is an odd number folding on $M^{\infty} \cap\left\{f^{\infty} \neq 0\right\}$, it implies that at least one of the interfaces has to bend in a wrong direction as they converge, which seems unlikely to happen. We would like to resolve these points in the future.

5.2. Implications to the Cahn-Hilliard equation. Consider a sequence of smooth initial data $u_{0}^{i}, i=1, \cdots$, and $\varepsilon_{i}$ with $\lim \sup E_{\varepsilon_{i}}\left(u_{0}^{i}\right)<$ $\infty, \varepsilon_{i} \rightarrow 0$. Suppose also that there exists a constant $m_{0} \in(-|U|,|U|)$ such that $\int_{U} u_{0}^{i}=m_{0}$ for all $i=\cdots$. Let $u^{i}$ be the solution to the Cahn-Hilliard equation (1.3) with the initial data. As noted, $f^{i}$ corresponding to $u^{i}$ satisfies

$$
\left.E_{\varepsilon_{i}}\left(u_{0}^{i}\right)=E_{\varepsilon_{i}}\left(u^{i}(\cdot, t)\right)+\int_{0}^{t} \int_{U}\left|\nabla f^{i}\right|^{2}\right)
$$

for all $t \geq 0$. Moreover, Chen proved that ([14, Lemma 3.4])

$$
\left\|f^{i}(\cdot, t)\right\|_{L^{2}(U)} \leq C\left(E_{\varepsilon_{i}}\left(u^{i}\right)+\left\|\nabla f^{i}(\cdot, t)\right\|_{L^{2}(U)}\right.
$$

holds for all $t$ and $i$ for all $\varepsilon_{i}$ small. Thus,

$$
\int_{0}^{t}\left\|f^{i}(\cdot, t)\right\|_{H^{1}(U)}^{2} \leq C
$$

for all large $i$. For $L^{1}$ a.e. $t$, we have $\lim \inf \left\|f^{i}(\cdot, t)\right\|_{H^{1}(U)}<\infty$ by Fatou's Lemma. We now restrict our attension to $n=2$ and such $t$. With a suitable growth condition on $W$ (such as $W \geq|u|^{k}, k>2$, for all large $u$ ) and the Neumann boundary condition, one may show that there exists a constant $c=c\left(U,\left\|f^{i}\right\|_{H^{1}(U)}\right)$ such that $\sup \left|u^{i}\right| \leq c$. Thus, for $n=2$, assumptions $\mathbf{A}$ and $\mathbf{B}$ are satisfied for a subsequence on this time slice. Unfortunately, even though we may conclude that there exists a subsequence for a.e. time slice for which we may apply our result, the choice of the subsequence may differ for each $t$. Note that the bound on $\left\|f^{i}(\cdot, t)\right\|_{H^{1}(U)}$ implies that the time derivative of the total energy stays finite, not allowing a violent jump of mass there. 


\section{YOSHIHIRO TONEGAWA}

\section{REFERENCES}

[1] W. Allard, On the first variation of a varifold, Ann. of Math. (2) 95 (1972) 417-491.

[2] S.M. Allen, J.W. Cahn, A macroscopic theory for antiphase boundary motion and its application to antiphase domain coarsening, Acta. Metal. 27 (1979) 1085-1095.

[3] N.D. Alikakos, P.W. Bates, X. Chen, Convergence of the Cahn-Hilliard equation to the Hele-Shaw model, Arch. Rational Mech. Anal. 128 (1994), no. 2, 165-205.

[4] N.D. Alikakos, L. Bronsard, G. Fusco, Slow motion in the gradient theory of phase transitions via energy and spectrum, Calc. Var. Partial Differential Equations 6 (1998), no. 1, 39-66.

[5] N.D. Alikakos, G. Fusco, The spectrum of the Cahn-Hilliard operator for generic interface in higher space dimensions, Indiana Univ. Math. J. 41 (1993), no. $2,637-674$.

[6] S. Baldo, Minimal interface criterion for phase transitions in mixtures of CahnHilliard fluids, Ann. Inst. H. Poincaré Anal. Non Linéaire 7 (1990), no. 2, $67-90$.

[7] P.W. Bates, E.N. Dancer, J. Shi, Multi-spike stationary solutions of the CahnHiliard equation in higher-dimension and instability, Adv. Diff. Eqns. 4 (1999), no. 1, 1-69.

[8] P.W. Bates, P.C. Fife, The dynamics of nucleation for the Cahn-Hilliard equation, SIAM J. Appl. Math. 53 (1993) 990-1008.

[9] P.W. Bates, G. Fusco, Equilibria with many nuclei for the Cahn-Hiliard equation, J. Diff. Eqns. 160 (2000), no. 2, 283-356.

[10] J.F. Blowey, C.M. Elliott, The Cahn-Hilliard gradient theory for the phase separation with non-smooth free energy, Part I: Mathematical analysis, European J. Appl. Math. 2 (1991) 233-279.

[11] J.W. Cahn, On the spinodal decomposition, Acta Metallurgy 9 (1961) 795-801.

[12] J.W. Cahn, J.E. Hilliard, Free energy of a non-uniform system I: Interfacial energy, J. Chem. Phys. 28 (1958) 258-267.

[13] X. Chen, Generation and propagation of interface in reaction-diffusion equations, J. Diff. Eqns. 96 (1992), no. 1, 116-141.

[14] X. Chen, Global asymptotic limit of solutions of the Cahn-Hilliard equation, J. Diff. Geom.44 (1996), no. 2, 262-311.

[15] X. Chen, D. Hilhorst, E. Logak, Asymptotic behavior of solutions of an AllenCahn equation with a nonlocal term, Nonlinear Anal. 28 (1997), no. 7, 12831298.

[16] E. De Giorgi, Convergence problems for functionals and operators, In Proc. Int. Meeting on recent methods in nonlinear analysis, eds. E. De Giorgi et al. Bologna: Pitagora (1979) 223-244.

[17] L.C. Evans, H.M. Soner, P.E. Souganidis, Phase transitions and generalized. motion by mean curvature, Comm. Pure Appl. Math. 45 (1992), no. 9, 10971123.

[18] L.C. Evans, R.F. Gariepy, Measure theory and fine properties of functions, Studies in Advanced Math., CRC Press, (1992).

[19] P.C. Fife, Nonlinear diffusive waves, CBMS Conf. Utah 1987, CBMS Conf. Series, (1989). 


\section{PHASE FIELD MODEL}

[20] I. Fonseca, L. Tartar, The gradient theory of phase transitions for systems with two potential wells, Proc. Roy. Soc. Edin. 111A (1989), no. 1-2, 89-102.

[21] D. Gilbarg, N. Trudinger, Elliptic partial differential equations of second order, Springer Verlag, Berlin, Heidelberg, New York, 1977.

[22] M.E. Gurtin, H. Matano, On the structure of equilibrium phase transitions within the gradient theory of fluids, Quart. App. Math. 46 (1988), no.2, 301317.

[23] M.E. Gurtin, Some results and conjectures in the gradient theory of phase transitions, In metastability and incompletely posed problems, eds. S. Antman et al. 301-317, Springer 1987.

[24] J. Hutchinson, Y. Tonegawa, Convergence of phase interfaces in the van der Waals-Cahn-Hilliard theory, Calc. Var. Partial Diff. Eqns. 10 (2000), no. 1, 49-84.

[25] T. Ilmanen, Convergence of the Allen-Cahn equation to Brakke's motion by mean curvature, J. Diff. Geom. 38 (1993), no. 2, 417-461.

[26] R. Kohn, P. Sternberg, Local minimizers and singular perturbations, Proc. Roy. Soc. Edin. 111A (1989), no. 1-2, 69-84.

[27] S. Luckhaus, L. Modica, The Gibbs-Thompson relation within the gradient theory of phase transitions, Arch. Rational Mech. Anal. 107 (1989), no. 1, 71-83.

[28] N. Meyers, W.P. Ziemer, Integral inequalities of Poincare and Wirtinger type for $B V$-functions, American Journal of Math. 99 (1977), 1345-1360.

[29] L. Modica, A gradient bound and a Liouville theorem for nonlinear Poisson equations, Comm. Pure Appl. Math. 38 (1985), no. 5, 679-684.

[30] L. Modica, Gradient theory of phase transitions and minimal interface criteria, Arch. Rational Mech. Anal. 98 (1987), no. 2, 123-142.

[31] L. Modica, Gradient theory of phase transitions with boundary contact energy, Ann. Inst. H. Poincaré Anal. Non Linéaire 4 (1987), no. 5, 487-512.

[32] W.W. Mullins, J. Sekerka, Morphological stability of a particle growing by diffusion or heat flow, J. Appl. Math. 34 (1963) 322-329.

[33] P. Padilla, Y. Tonegawa, On the convergence of stable phase transitions, Comm. Pure Appl. Math. 51 (1998), no. 6, 551-579.

[34] R. Pego, Front migration for the nonlinear Cahn-Hilliard equation, Proc. Roy. Soc. Lond. 422 (1989) 261-278.

[35] N. Owen, Nonconvex variational problems with general singular perturbations, Trans. Amer. Math. Soc. 310 (1988), no. 1, 393-404.

[36] R. Schätzle, Hypersurfaces with mean curvature given by a trace, to appear in J. Diff. Geom.

[37] R. Schätzle, Quadratic tilt-excess decay and strong maximum principle for varifolds, preprint.

[38] L. Simon, Lectures on geometric measure theory, Proc. Centre Math. Anal. Austral. Nat. Univ. 3 (1983).

[39] P. Sternberg, The effect of a singular perturbation on nonconvex variational problems, Arch. Rational Mech. Anal. 101 (1988), no. 3, 209-260.

[40] P. Sternberg, Vector-valued local minimizers of nonconvex variational problems, Rocky Mountain J. Math. 21 (1991), no. 2, 799-807.

[41] B. Stoth, Convergence of the Cahn-Hilliard equation to the Mullins-Sekerka problem in spherical symmetry, Comm. Partial Diff. Eqns. 125 (1996), no. 1, 154-183. 


\section{YOSHIHIRO TONEGAWA}

[42] J. Wei, M. Winter, Multi-interior-spike solutions for the Cahn-Hilliard equation with arbitrary many peaks, Cal. Var. Partial Diff. Eqns. 10 (2000), no. 3, 249-289.

[43] J. Wei, M, Winter, On the stationary Cahn-Hilliard equation: interior spike solutions, J. Diff. Eqns. 148 (1998), no. 2, 231-267.

[44] W.P. Ziemer, Weakly differentiable functions, Springer Verlag (1989).

Department of Mathematics, HokKaido University, Sapporo 060-0810, JAPAN

E-mail address: tonegawa@math.sci.hokudai.ac.jp 